\title{
Synchronization in two neurons: Results for a two-component dynamical model with time-delayed inhibition
}

\author{
Gilles Renversez ${ }^{1}$ \\ Centre de Physique Théorique, UPR 7061 du Centre National de la Recherche Scientifique, Campus de Luminy, Case 907, \\ 13288 Marseille Cedex 9, France
}

Received 17 December 1996; received in revised form 21 May 1997; accepted 23 June 1997

Communicated by Y. Kuramoto

\begin{abstract}
We study synchronization in two neurons using two-dimensional neuronal models coupled through threshold delayed inhibition, acting for a fixed duration $T$. We explain the mechanism of synchronization between the two coupled units by means of phase-plane analysis. Using a piecewise linear approximation, we compute the firing times of two mutually coupled neurons, which allows a quantitative study of synchronization. The results are in good agreement, both quantitatively and qualitatively, with the numerical simulations of the full nonlinear coupled system describing the two neurons. We first show that, with simple inhibition, only phase-lagged synchronization can be achieved except for a particular set of $T$-values. We then show that coincidence synchronization can rapidly be achieved with an improved coupling model which takes into account the increase of membrane conductance during inhibition. Copyright $@ 1998$ Elsevier Science B.V.
\end{abstract}

PACS: 02.30.Ks; 05.45.+b; 87.10.+e

Keywords: Neuronal modelling; Synchronization; Inhibition; Time-delayed coupling; Phase-plane analysis

\section{Introduction}

Synchronization of coupled oscillators has been studied as long ago as 1665 by Huygens. In spite of the great amount of work on this subject, it has gained a new interest with its possible application to biological neural networks.

The key role of time delays in biological neuronal networks was previously mentioned by Andersen et al. [3] to explain the postsynaptic inhibition exerted on hippocampal pyramidal neurons. In a more recent study of hippocampal slices, Miles et al. [25] reported the observation of monosynaptic EPSPs excitatory postsynaptic potentials latencies ranging from 2.5 to approximately $8.5 \mathrm{~ms}$. Time delays are also present in other vertebrate neuronal strutures like the opossum prepyriform cortex [15] or the visual system (see [29] for a comprehensive review). In the case of invertebrates, time delays are also required to understand neural circuits such as the flight system of the locust [34] or the sound localization system of the owl [9]. Time delays are due to both finite propagation

\footnotetext{
${ }^{1}$ Universités Paris XI Orsay et Aix-Marseille I. Fax: +33 49126 9553; e-mail: renverse @ cpt.univ-mrs.fr.
} 
velocities in the conduction of signals between neurons in the neurites [23] and delays in the synaptic transmission at chemical synapses [20]. Still from a biological point of view, the role of inhibitory neurons has been re-evaluated [8] (see also [7, Chs. 34 and 35]): the role of inhibition in the cortex is not solely to ensure the stability of neuronal activity. From a theoretical point of view, time delays may account for a significant percentage of the interspike interval and hence completely modify network dynamics, as has been shown for phase oscillators [27,35] and for leaky integrate-and-fire neurons [28]. For both these models [28,40], and also for pulse-coupled oscillators [11], it has been demonstrated that one should include inhibition to achieve synchronization with biologically plausible coupling, i.e., with non-instantaneous or delayed interactions.

The aim of the present paper is to get closer to biological neurons in the study of synchronization, compared with other works. Nevertheless, we still want to keep an analytical control on the results. The phase description for neuron models, which allows to study synchronization mechanisms in detail even within fully connected networks [18], will not be used hereafter because it is valid only at weak coupling [17]. Moreover, as pointed out by Somers and Kopell [36,37] phase-type two-component neuron models have a different behaviour in terms of synchronization than the same models in a relaxation-type regime. Thus, a two-component model in the relaxation-type regime will be used. This kind of model incorporates both the effects of cell capacitance and of voltage-dependent membrane conductances [22,24,30], whereas leaky integrate-and-fire model does not take into account the latter [1]. Besides, two-component models can be studied in detail by means of phase-plane analysis [32]. Therefore, a neuron will be regarded as a particular type of oscillator, a Terman-Wang unit [38] in which two variables describe the neuron's dynamics, one being potential-like and the other a recovery variable. It has been shown in [38] that this type of oscillator locally connected through zero-delay excitatory coupling is able to achieve synchronization within blocks of oscillators which are stimulated by connected regions. However, previous studies $[36,38]$ do not include the effects of transmission delays. In the present paper, the synchronization between two mutually coupled twocomponent neuron models interacting through time-delayed inhibition will be studied, with the motivation that the understanding of the behaviour of two mutually coupled neurons is, in several cases, the starting point or the guideline for the study of more complete networks $[11,28]$.

Another important difference from previous studies is that the coupling we consider is a threshold coupling acting during a fixed duration, and not an instantaneous coupling, as in pulse-coupled oscillators [11], or a coupling acting only during the spike duration as in [36,38].

In what follows, we shall briefly recall the main properties of the neuron model used in Section 2. In Section 3, it will be shown that, with a simple delayed inhibition acting only on the potential-like variable, only phase-lagged synchronization can be achieved except for the case of a particular set of values of the duration of the inhibition. This will be shown with the help of a piecewise linear model, in the phase space, of the full nonlinear system describing the neuron. The observed behaviour of the nonlinear system is explained in the framework of the piecewise linear model. Then, in Section 4, it will be shown, with the same methods, that coincidence synchronization may be reached rapidly with a more complete coupling taking into account the increase of membrane conductance during neurophysiological inhibition. Finally, the results will be discussed in Section 5 and compared with those obtained with other models.

\section{Model for a single neuron}

Each neuron is described by a set of two nonlinear coupled equations of the form [38]:

$$
\begin{aligned}
& \dot{v}=-v^{3}+3 v+2-u+I_{v}+E_{v}, \\
& \dot{u}=c(\gamma(1+\tanh (v / \beta))-b u),
\end{aligned}
$$




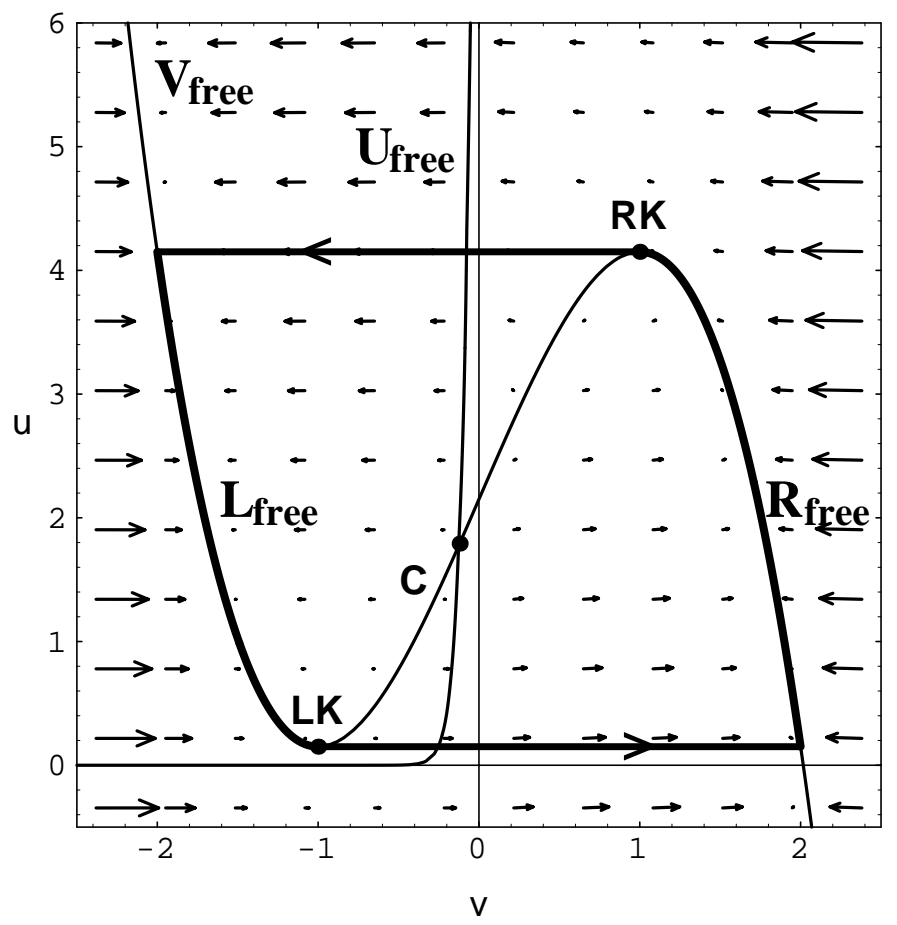

Fig. 1. $u$-nullcline, $\mathcal{U}_{\text {free }}, v$-nullcline, $\mathcal{V}_{\text {free }}$, and vector fields for one uninhibited stimulated Terman-Wang unit (thin lines). $\mathcal{L}_{\text {free }}$ and $\mathcal{R}_{\text {free }}$ (thick lines) are parts of $\mathcal{V}_{\text {free }}$, together with the two segments at constant $u$, they represent the "singular limit" trajectory in the phase plane. The two arrows indicate the direction of motion. $C$ is an unstable fixed point, LK and RK are defined in the text.

$v$ is a potential-like variable and $u$ a recovery-like variable which governs the repolarizing conductance. $I_{v}$ is the coupling with the other unit (non-null only during the inhibition duration), and $E_{v}$ is an external stimulation which will be taken constant. The spiking neuron model described by Eqs. (1) and (2) is a point model. This approximation is justified when inhibitory coupling is considered because inhibitory synapses are mainly positioned on cell somata $[2,6,14]$.

The $v$-nullcline $\mathcal{V} \equiv\{(v, u)$ such that $\dot{v}=0\}$ is a cubic curve while the $u$-nullcline $\mathcal{U} \equiv\{(v, u)$ such that $\dot{u}=0\}$ is a sigmoid function. We denote by $\mathcal{R}$ the right branch of $\mathcal{V}$ and $\mathcal{L}$ its left branch. We denote by LK (respectively $\mathrm{RK}$ ) the lower knee of $\mathcal{L}$ (respectively the upper knee of $\mathcal{R}$ ). In what follows, the parameter $c$ will always be taken smaller than one in both the analytic approach and the computer simulations. The analytic approach will deal with "singular solutions" $(c \rightarrow 0)$, i.e., with the limiting case in which the jumps between branches take place infinitely fast [13].

The unit will be said stimulated if $E_{v}>0$ and unstimulated for $E_{v}<0$. Stimulated non-coupled units are oscillatory whereas unstimulated units do not oscillate (no action potential is emitted). In the stimulated case, we denote by $\mathcal{R}_{\text {free }}$ the right branch of $\mathcal{V}_{\text {free }}$ and $\mathcal{L}_{\text {free }}$ its left branch (see Fig. 1). The knees, or extrema of the cubics, represent thresholds for jumping to the other branch. Thus each unit in the relaxation regime has an onset threshold at the local minima and an offset threshold at the local maxima. The orbital trajectory in the phase plane is therefore composed of one segment on $\mathcal{L}_{\text {free }}$ and an adjacent segment on $\mathcal{R}_{\text {free }}$ for the slow part of the trajectory bound together with instantaneous jumps at constant $u$ (see Fig. 1). It may easily be seen, from the shape of $\mathcal{U}_{\text {free }}$, that the depolarization duration is much longer than the action potential duration, and that their ratio increases with $\gamma$. 


\section{A system of two units with inhibitory coupling in the $v$ component}

\subsection{Threshold coupling with a fixed period}

"Threshold coupling" acts on the postsynaptic unit at time $t$ only if the presynaptic potential is above a fixed threshold at time $t-\Delta$ where $\Delta$ is the coupling delay (for non-delayed coupling as considered in [36,38], one has $\Delta=0$ ). With this definition, the coupling duration is equal to the spike duration. This kind of coupling is based on action potential occurrence, which is known to be essential in order to process information [12,39]. However, it still does not take into account neurophysiological data on the duration of EPSPs and IPSPs which are known to last longer than the spike (especially with IPSPs) [21]. Hence, these models go one step further than pulse-coupled models but can still be improved in managing the effects of the coupling period on the postsynaptic neuron.

In the present model, the same definition will be used for the coupling onset which occurs at threshold, but the coupling duration, $T$, will be allowed to differ from the spike duration. We shall limit our study to the case where the coupling delay, $\Delta$, is smaller than the interspike intervals of the stimulated unit and where the postsynaptic neuron receives a single action potential from the presynaptic neuron during an interspike interval.

In biological spiking processes, the action potential amplitude and duration do not depend on the coupling. Therefore, in order to keep biological relevance, the interaction between units should not modify the spike amplitude and duration which is possible (see [38, Fig. 10]) with two variables relaxation type neuron model using nonconditional coupling in the postsynaptic unit. Thus the interaction does not act during the spiking process, which excludes the phase-space region corresponding to fast evolutions and portions of $\mathcal{R}$ of postsynaptic dynamics.

We shall assume in this section that the inhibition makes $I_{v}$ negative in Eq. (1) only throughout its duration. This term $I_{v}$ corresponds to the usual hyperpolarizing current present during inhibition in leaky integrate-and-fire models. In the phase-space, the $u$-nullcline is the same with or without inhibition, i.e., $\mathcal{U}_{\text {free }}$, and only the $v$-nullcline is modified. This latter is just translated downward as long as inhibition is active, it will be denoted by $\mathcal{V}_{\text {inhib }}$ (see Fig. 2). As in Section 2, $\mathcal{L}_{\text {inhib }}$ will be defined as the left branch of $\mathcal{V}_{\text {inhib. }}$

With this coupling acting only on variable $v$, the point representing the receiving unit is always above $\mathcal{U}_{\text {free }}$ when it gets inhibition. Its trajectory in phase-space is then the following: before inhibition it moves along $\mathcal{L}_{\text {free }}$, then, during a relatively short period, it moves from $\mathcal{L}_{\text {free }}$ to $\mathcal{L}_{\text {inhib }}$.

This results from the existence of two different timescales in the system defined by Eqs. (1) and (2). There is a factor $c$ between them and, since $c \ll 1$ (in most of our simulations $c=0.04)|(\mathrm{d} u / \mathrm{d} t) /(\mathrm{d} v / \mathrm{d} t)| \simeq 0$ except on the $v$-nullcline. Therefore, the trajectory between the two $v$-nullclines roughly follows a line of constant $u$. The situation is of the same type as the one encountered at spike onset where the starting point in phase-space is unstable and the trajectory follows a line of constant $u$ to join the curve corresponding with the associated limit solution [13] (in our case, this curve is $\mathcal{L}_{\text {inhib }}$ and, in [13], it is $\mathcal{R}_{\text {free }}$ ). When the unit's position is on $\mathcal{L}_{\text {inhib }}$, it moves toward the stable fixed point $S^{\prime}$ corresponding to the intersection of $\mathcal{L}_{\text {inhib }}$ and $\mathcal{U}_{\text {free }}$. This stable fixed point exists because: (i) with the considered inhibition, $\mathcal{V}_{\text {inhib }}$ is just translated downward compared to $\mathcal{V}_{\text {free }}$, and (ii) the inhibition intensity was chosen such as to ensure the existence of the intersection of $\mathcal{L}_{\text {inhib }}$ and $\mathcal{U}_{\text {free }}$.

Since, on $\mathcal{L}_{\text {inhib }}, u$ is a monotically decreasing function of $v$, and since $S^{\prime}$ is below the knee of $\mathcal{L}_{\text {free }}, S^{\prime}$ is below any point which could be reached by a transient trajectory between the two $v$-nullclines (see Fig. 2). When inhibition is removed, the system is again described by Eqs. (1) and (2) with $I_{v}=0$. The unit rejoins $\mathcal{L}_{\text {free }}$ or it emits a spike, according to its position in phase space at the time the inhibition is removed.

To advance or delay the emission of an action-potential in an absolute way is not the way synchronization can be achieved. One should rather reduce the absolute time interval between the spikes of the two units. Hence, the lag induced by the coupling should depend on the position of the postsynaptic unit in the phase plane: the nearer to 


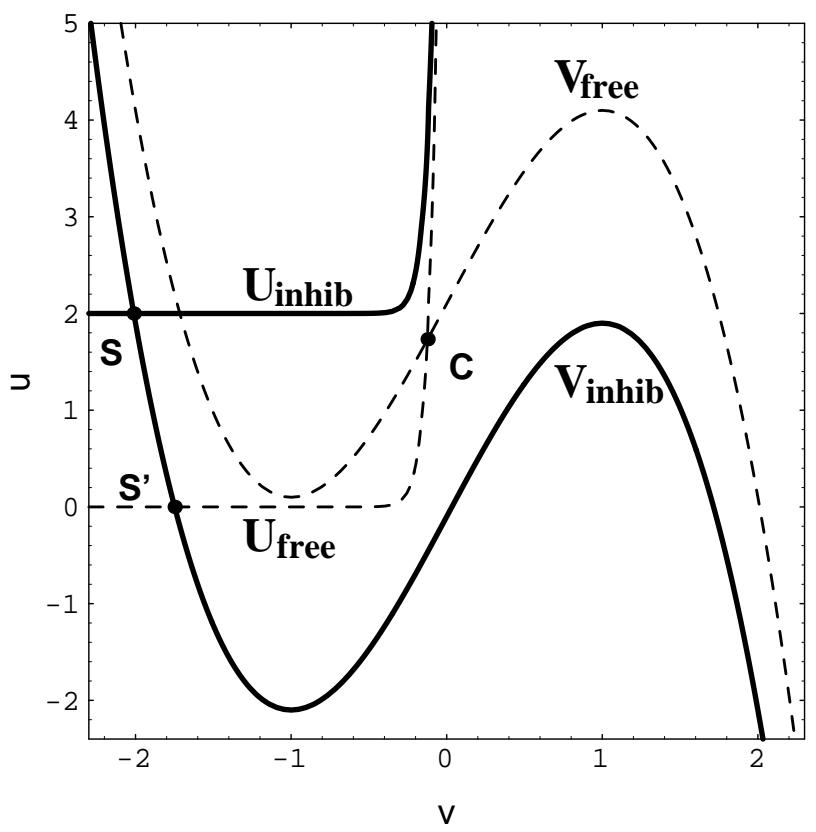

Fig. 2. Phase plane. For an uninhibited unit: the $v$-nullcline is $\mathcal{V}_{\text {free }}$ and the $u$-nullcline is $\mathcal{U}_{\text {free }}$. For the $v$-only inhibitory coupling: the $v$-nullcline is $\mathcal{V}_{\text {inhib }}$ (thick line), the $u$-nullcline is just $\mathcal{U}_{\text {free }}$ (dashed line), and $S^{\prime}$ is the associated stable fixed point. For the $u-v$ coupling: the $v$-nullcline is $\mathcal{V}_{\text {inhib }}$ (thick line), the $u$-nullcline is $\mathcal{U}_{\text {inhib }}$ (thick line), and $S$ is the associated stable fixed point.

spiking the unit is, the longer the induced lag must be. If the lag is independent of the location in the phase plane of the postsynaptic neuron, the two units will not reach impulse synchrony.

Let us estimate the effect of delayed inhibition in this model neglecting the transient periods corresponding to the fast transitions from one $v$-nullcline to another. The inhibition duration is fixed and we call it $T$. We have three cases to study:

(i) No unit reaches the stable fixed point of the inhibited system during the inhibition duration $T$, i.e., both units will rejoin $\mathcal{L}_{\text {free }}$ before spiking:

During $T$, the unit is described by Eqs. (1) and (2) with $I_{v}<0$ and hence it evolves on $\mathcal{L}_{\text {inhib. }}$ The same thing occurs for the others unit when it receives inhibition during the same duration $T$ (but not at the same moment). Since $I_{v}$ is independent of postsynaptic unit position in phase plane, the two units evolve during $T$ according to the same equations. The dynamics on $\mathcal{L}_{\text {inhib }}$ is then controlled by the $u$-equation (2) where the term $c \gamma(1+\tanh (v / \beta))$ is negligible. Therefore, while both units rejoin $\mathcal{L}_{\text {free }}$, inhibition modified $v$ time course during $T$ but at the end of the inhibition $v$ recovers the value it would have without inhibition. Thus, in this case the effect of inhibitory only $v$-coupling is null in terms of impulse synchronization: the coupling freezes the spiking times interval of the two units leading to phase-lagged synchronization (see Fig. 3).

(ii) Only the leading unit reaches the stable fixed point $S^{\prime}$, consequently this unit will emit a spike as soon as inhibition will be released and the lagged one will rejoin $\mathcal{L}_{\text {free }}$ (which implies that it will spike later):

The second unit is not delayed by the inhibition process as in the previous case, while the leading unit is delayed. This will reduce the time gap between the two units. In this case, the $v$-only coupling is synchronizing (see Fig. 3). We shall quantify this effect on the next section with a piecewise linear approximation of the full nonlinear system. 


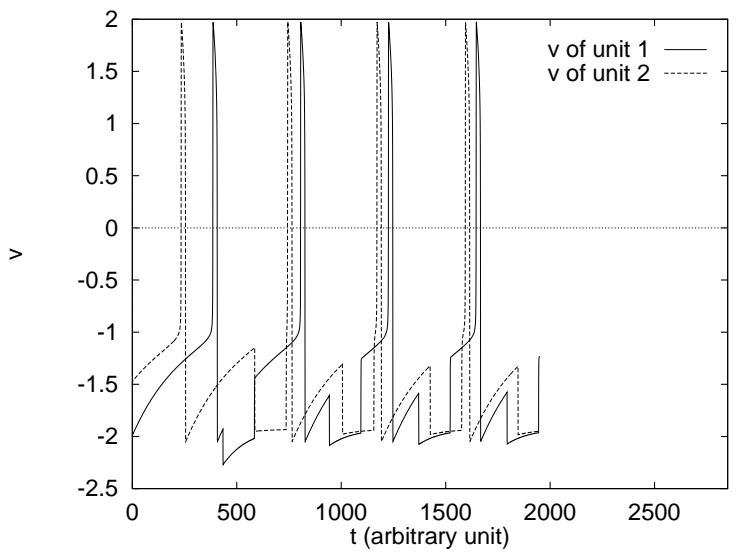

(a) $v$ versus time

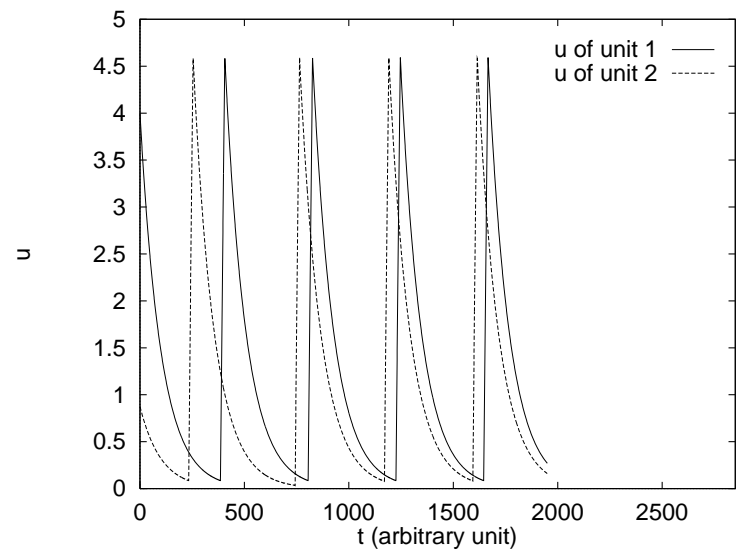

(b) $u$ versus time

Fig. 3. Example of a phase-lagged synchronization in two units with inbibitory delayed coupling in $v$. The first interaction corresponds to the case (ii) and the following ones to the case (i) $\left(c=0.04, \gamma=3.0, b=0.25, \beta=0.1, E_{v}=0.1, I_{v}=-2.2, T=150, \Delta=200\right)$.

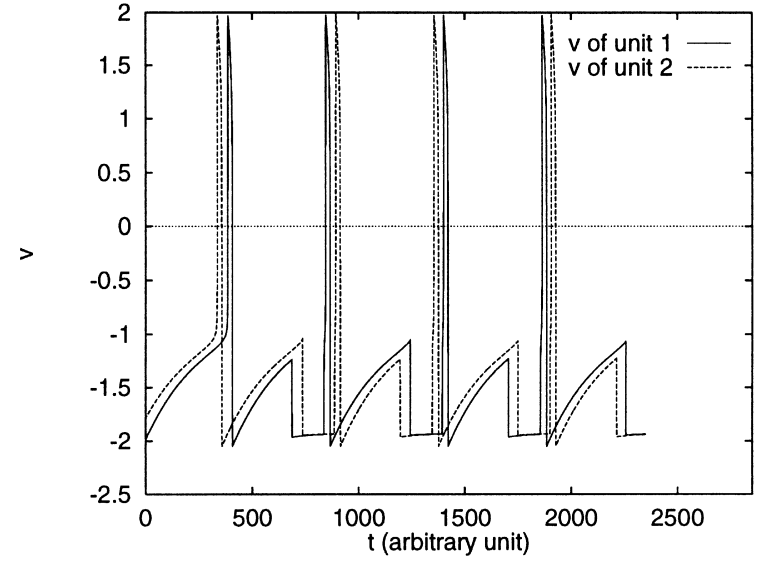

(a) $v$ versus time

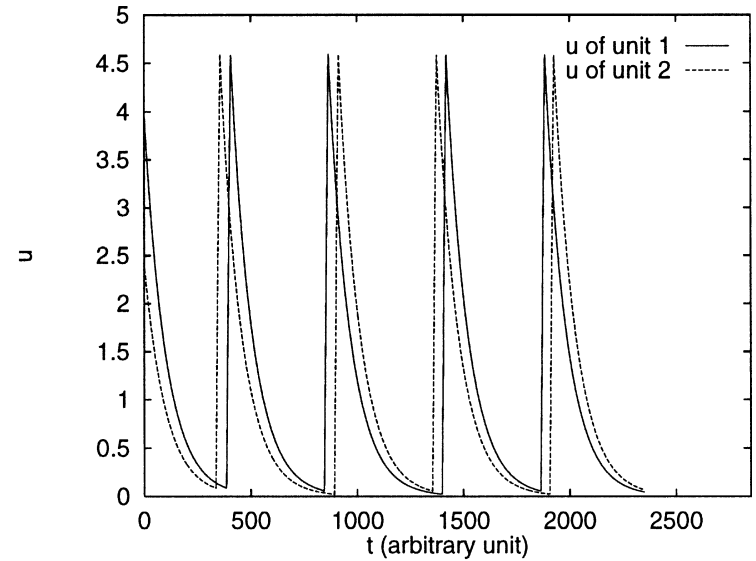

(b) $u$ versus time

Fig. 4. Example of order reversals in two units with inbibitory delayed coupling in $v$. The successive interactions correspond to the case (iii) $\left(c=0.04, \gamma=3.0, b=0.25, \beta=0.1, E_{v}=0.1, I_{v}=-3.5, T=150, \Delta=350\right.$. $)$

(iii) Both units reach the stable fixed point during the inhibition duration, consequently both units will emit a spike as soon as inhibition is released:

The lagged unit will receive inhibition from the leading unit and will be locked at the stable fixed point at least during a certain period. As already stated, this unit will emit a spike as soon as inhibition is released. On the other hand, inhibition on the leading unit will be released later since the coupling comes from the lagged unit, thus the latter is now the leading unit. We have here a spiking order reversal. As coupling terms will be the same as before and as they will act relatively at the same location in phase space, the units' roles will just be interchanged (see Fig. 4). In this case, we can prove the order reversal and we can compute easily the successive interspike intervals.

Let $t_{1}$ be the first spike time of unit $1, t_{2}$ the first spike time of unit 2 with $t_{1}<t_{2}$, and $\Delta$ the coupling delay. At $t=t_{1}+\Delta$ unit 2 is inhibited and at $t=t_{2}+\Delta$ unit 1 is inhibited. Both units fire when their inhibition is released. 
Thus, we have, for $t_{2}^{\prime}$ and for $t_{1}^{\prime}$, the second firing times of each unit:

$$
t_{1}^{\prime}=t_{2}+\Delta+T \text { and } t_{2}^{\prime}=t_{1}+\Delta+T .
$$

Since $t_{1}<t_{2}, t_{1}^{\prime}>t_{2}^{\prime}$. The unit 2 is now the leading unit and the units' roles are just interchanged. Order reversal will occur again at next firing. Let us note by $P_{+}$and $P_{-}$the two successive interspike intervals and define $\Delta t=t_{2}-t_{1}$, then one has

$$
P_{+}=\Delta+T+\Delta t \quad \text { and } \quad P_{-}=\Delta+T-\Delta t .
$$

\subsection{Piecewise linear model in the phase plane}

In order to get further analytical insights into synchronization mechanisms, we shall define, in the phase plane, a piecewise linear model of the system defined by Eqs. (1) and (2). In [33], Rinzel and Keller used a similar approach for the Fitzhugh-Nagumo model, but without considering any coupling. Let us first explain how we build each linear part of this model, which is only valid during the repolarization period. We want to linearize the $v$-terms on the r.h.s. of Eq. (1), let us define $f(v)$ as equal to $-v^{3}+3 v+2$. Let us choose a point $Q\left(U_{Q}, V_{Q}\right)$ such as $V_{Q} \in[-2,-1]$, and since we only consider the repolarization period, we take the tangent at $Q$ to $f(v)$. We denote its slope by $k=f^{\prime}\left(V_{Q}\right)$ and by $l$ the value of $f\left(V_{Q}\right)$. In the case of the $u$-nullcline, we can neglect the factor $c \gamma(1+\tanh (v / \beta))$ since it is nearly equal to zero all along the $v$-interval corresponding to the repolarization region, the main term being $-c b u$. Thus, one gets

$$
\begin{aligned}
& \dot{v}=k\left(v-V_{Q}\right)+l-u+I_{v}+E_{v}, \\
& \dot{u}=-c b u .
\end{aligned}
$$

Let us now define $V_{\text {th }}$ as the threshold potential for action potential emission which is such as $k\left(V_{\text {th }}-V_{Q}\right)+l=0$ and corresponds to the point LK in the "free" nonlinear system. We shall limit our study to the case where $-I_{v}>E_{v}$ so as to ensure the existence of a fixed point $S^{\prime}$ with $V_{S^{\prime}}<V_{\text {th }}$ during inhibition duration. We can rewrite the above system using linear matrix formalism since now it is piecewise linear:

$$
\begin{aligned}
& \frac{\mathrm{d} \mathbf{u}(t)}{\mathrm{d} t}=\mathbf{M u}(t)+\mathbf{a}, \quad \text { where } \mathbf{u}(t)=(u(t) \quad v(t))^{\mathrm{t}}, \\
& \mathbf{M}=\left(\begin{array}{cc}
-b c & 0 \\
-1 & k
\end{array}\right), \\
& \mathbf{a}=\left\{\begin{array}{cl}
\mathbf{a}_{\mathrm{i}}=\left(\begin{array}{c}
0 \\
-k V_{Q}+l+I_{v}+E_{v}
\end{array}\right) & \text { if the system is "inhibited", } \\
0 & \text { if the system is "free". }
\end{array}\right.
\end{aligned}
$$

We shall now compute the time interval between spikes in each unit after an interaction according to the one before, when no spike is suppressed. By interaction, we mean that both units receive the inhibitory coupling from the other. Thus there are the following three steps between the two spikes of a same unit:

(i) Suppose that at $t=t_{1}$, unit 1 emits a spike and that at $t=t_{2}$, unit 2 emits a spike, with $t_{2}-t_{1}=\Delta t>0$. At $t=t_{1}+\Delta$, unit 2 receives the coupling from unit 1 (where $\Delta$ is defined as previously as the coupling delay). Thus, in the time interval $\left[t_{2}, t_{1}+\Delta\right]$, unit 2 is described by the "free" system, while in the interval $\left[t_{1}, t_{2}+\Delta\right]$, unit 1 is described by the "free" system. 


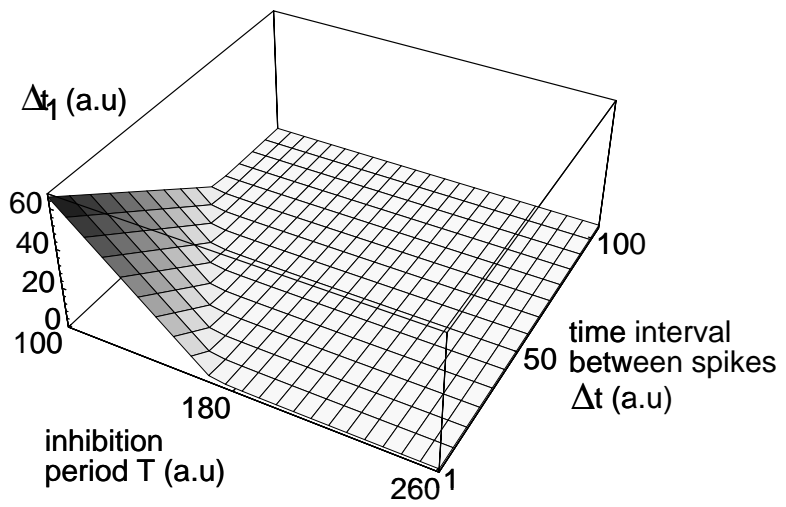

Fig. 5. Time before spiking, $\Delta t_{1}$, after release from inhibition for unit 1 in the piecewise linear model plotted against inhibition period $T$ and time interval $\Delta t$ between the spikes of the two units $\left(\Delta=165, E_{v}=0.15, I_{v}=-3.5, b\right.$ and $c$ are the same as in Fig. 3).

(ii) At $t=t_{1}+\Delta$, the inhibition begins for unit 2; it will end at $t=t_{1}+\Delta+T$ (where $T$ is defined as previously as the inhibition duration). Thus, during this interval $\left[t_{1}+\Delta, t_{1}+\Delta+T\right]$, unit 2 is described by the inhibited system, and similarly, during the interval $\left[t_{2}+\Delta, t_{2}+\Delta+T\right]$, unit 1 is described by the inhibited system.

(iii) At $t=t_{1}+\Delta+T$, the inhibition for unit 2 ends. We denote by $\Delta t_{2}$, the time then required by unit 2 to reach the spiking threshold $V_{\text {th }}$ (seeing that the inhibited system cannot spike). Thus, during the interval $\left[t_{1}+\Delta+T, t_{1}+\Delta+T+\Delta t_{2}\right]$, unit 2 is described by the "free" system. Similarly, denoting by $\Delta t_{1}$, the time required by unit 1 after the inhibition end to reach the spiking threshold, during $\left[t_{2}+\Delta+T, t_{2}+\Delta+T+\Delta t_{1}\right]$, unit 1 is described by the "free" system.

Let us denote by $t_{1}^{\prime}$ and $t_{2}^{\prime}$ the respective firing times of the two units. We can define a synchronization rate, $\sigma_{\mathrm{s}}$, as the ratio between the successive differences of the two units spiking times:

$$
\sigma_{\mathrm{s}}=\frac{t_{2}^{\prime}-t_{1}^{\prime}}{t_{2}-t_{1}}
$$

Then, since $t_{1}^{\prime}=\Delta t_{1}+\Delta+T+t_{2}, t_{2}^{\prime}=\Delta t_{2}+\Delta+T+t_{1}$, and $\Delta t^{\prime}=t_{2}^{\prime}-t_{1}^{\prime}=\left(\Delta t_{2}-\Delta t_{1}\right)-\Delta t$, one gets

$$
\sigma_{\mathrm{s}}=\frac{\Delta t^{\prime}}{\Delta t}=\frac{\Delta t_{2}-\Delta t_{1}}{\Delta t}-1
$$

The detailed calculations and the expressions used to compute $\Delta t_{1}$ and $\Delta t_{2}$ are given in Appendix A.1. The results are shown in Fig. 5 for $\Delta t_{1}$, in Fig. 6 for $\Delta t_{2}$, and in Fig. 7 for $\sigma_{\mathrm{s}}$.

The plateau associated with $\Delta t_{1}$ in Fig. 5 corresponds to the freezing of unit 1 in $S^{\prime}$, i.e., in this case the actionpotential is fired as soon as the inhibition is removed. In a similar way, the plateau associated with $\Delta t_{2}$ in Fig. 6 corresponds to the freezing of the unit 2 in $S^{\prime}$. The combination of these two situations gives the case (iii) of Section 3.1. There is an order reversal of the spike times without any decrease of the absolute value of the time intervals between the action potentials of the two units (see, in Fig. 7, the region where $\sigma_{\mathrm{s}}$ is equal to -1 ).

The varying part of $\Delta t_{1}$ in Fig. 5 corresponds to the situation where unit 1 does not reach $S^{\prime}$ during inhibition. In a similar way, for unit 2, the varying part of $\Delta t_{2}$ in Fig. 6 corresponds to the situation where unit 2 does not reach $S^{\prime}$ during inhibition. The combination of both these situations gives the case (i) of Section 3.1. There is a freezing of the spiking times interval of the two units (see, in Fig. 7, the corresponding region where $\sigma_{\mathrm{s}}$ is equal to 1 ).

In Fig. 7, the synchronization rate is plotted versus inhibition duration, $T$, and time interval between the spikes of the two units, $\Delta t$. For small $\Delta t$ in Fig. 7, the transition from 1 to -1 regions is very steep. A fixed system evolutes at constant $\Delta$, therefore except for the rare case where it finaly falls into the transition region, an initial situation 


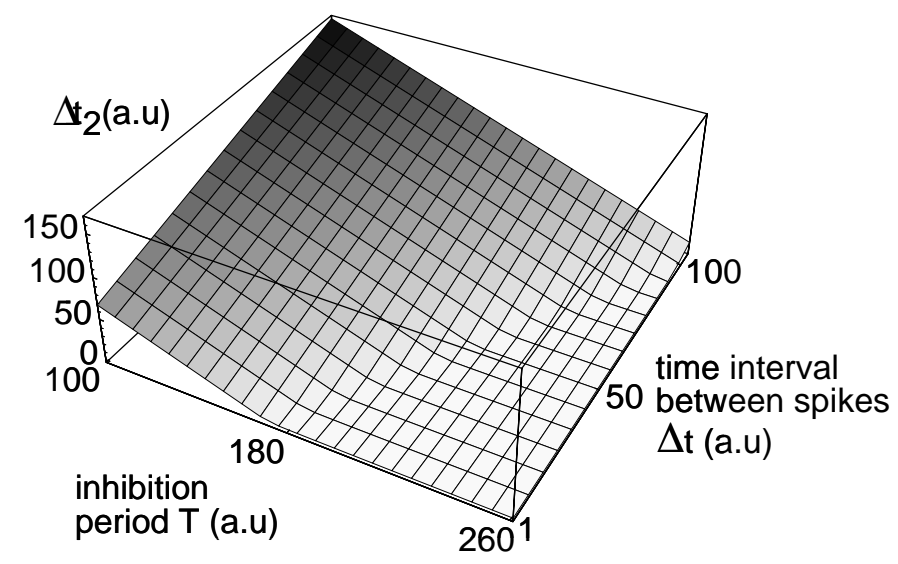

Fig. 6. Time before spiking, $\Delta t_{2}$, after release from inhibition for unit 2 with the piecewise linear model plotted against inhibition period $T$ and time interval $\Delta t$ between the spikes of the two units (the parameters are the same as in Fig. 5).

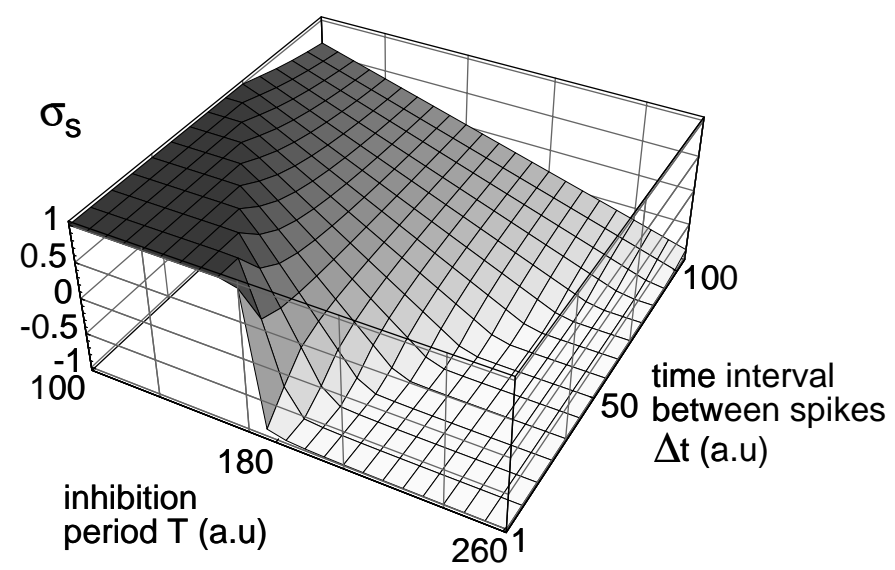

Fig. 7. Synchronization rate $\sigma_{\mathrm{s}}$ for the piecewise linear model plotted against inhibition period $T$ and time interval $\Delta t$ between the spikes of the two units (the parameters are the same as in Fig. 5).

corresponding to the case (ii) leads in a few steps to either the case (i) $\left(\sigma_{\mathrm{s}}=1\right)$ or the case (iii) $\left(\sigma_{\mathrm{s}}=-1\right)$ of Section 3.1. This is exactly what is shown in Fig. 3.

It must be noted that the position of the region where the coupling is synchronizing depends on the value of external stimulation $E_{v}$, so that a coincidence synchronization occurring for a fixed value of $E_{v}$ cannot be warranted to hold for another value of $E_{v}$.

In Fig. 8, one may see that the results obtained from the piecewise linear approximation of the full nonlinear system rather agree, both qualitatively and quantitatively, with those obtained numerically from the full model. The agreement is better than $10 \%$. The discrepancy comes from the nonlinear terms which are neglected in the piecewise linear model.

In order to be more biologically relevant, we now take into account the linear dependency on postsynaptic potential in the coupling term [16]. We take $I_{v}=\left(v-V_{\text {rev }}\right) R_{v}$, where $V_{\text {rev }}$ is similar to a Nernst potential associated with 


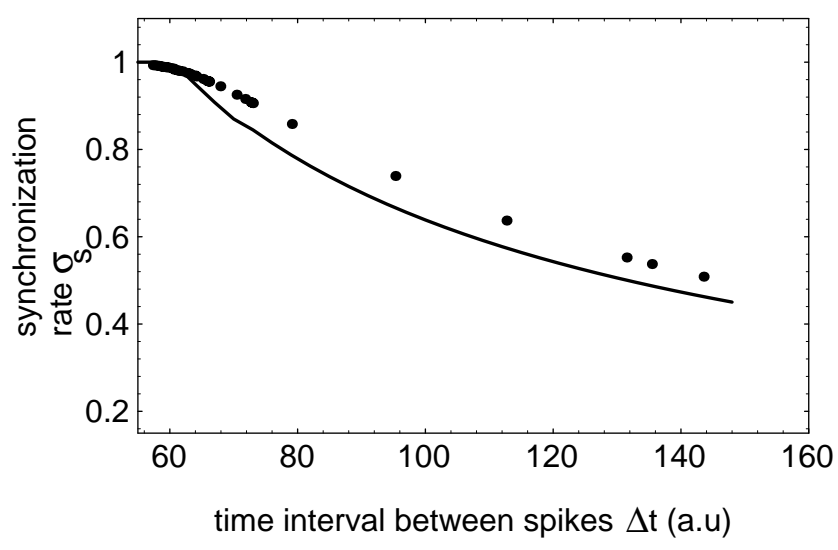

Fig. 8. Comparison between the synchronization rate, $\sigma_{\mathrm{s}}$, obtained from the numerical results (dots) for a system of two neurons with a $v$ inhibitory-type coupling, with those obtained from analytical results (solid line) with the piecewise linear model $(T=156, \Delta=165$, $E_{v}=0.1, I_{v}=-3.5, b$ and $c$ are the same as in Fig. 3).

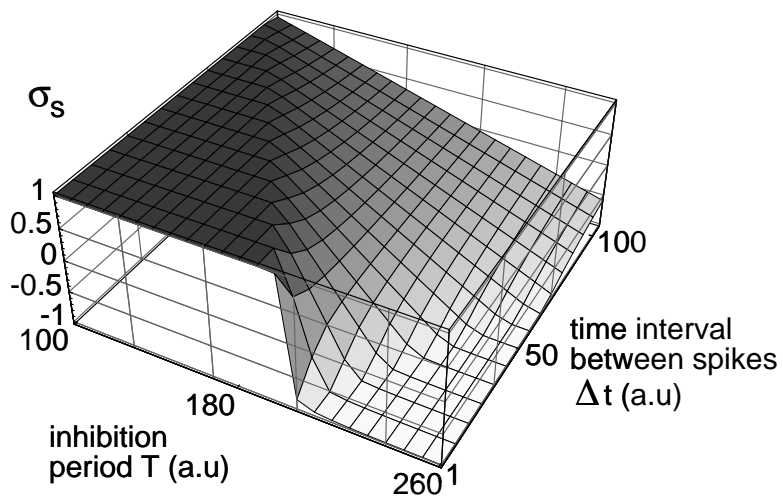

Fig. 9. Synchronization rate $\sigma_{\mathrm{s}}$ for the piecewise linear model, for a $v$-only coupling with a linear dependency on postsynaptic potential, plotted against inhibition period $T$ and time interval $\Delta t$ between the spikes of the two units $\left(\Delta=165, E_{v}=0.15, R_{v}=-2.0, V_{s}=-1.8\right.$, $b$ and $c$ are the same as in Fig. 3).

ions responsible for inhibition. By means of the same kind of calculus as the ones used for a constant coupling (see Appendix A.2), we can compute the synchronization rate $\sigma_{\mathrm{s}}$. The results are similar to those obtained from the constant coupling on variable $v$ as one can see in Fig. 9.

The synchronization rate as a function of the coupling strength $R_{v}$ (respectively of the reversal potential $V_{\mathrm{s}}$ ) is plotted in Fig. 10 (respectively in Fig. 11). For these two figures, the synchronization rate is small since we choose $\Delta$ and $T$ values so as to avoid parameter values where $\sigma_{\mathrm{s}}$ is equal to \pm 1 . In Fig. 12, the synchronization rate is shown as a function of the coupling delay $\Delta$. The same behaviour occurs with the constant $v$-only coupling. This change of the slope of $\sigma_{\mathrm{s}}$ corresponds to the limit case where the first unit reaches the stable fixed point $S^{\prime}$. The $\Delta_{\text {limit }}$ corresponding to this situation is approximated in Appendix A.1. 


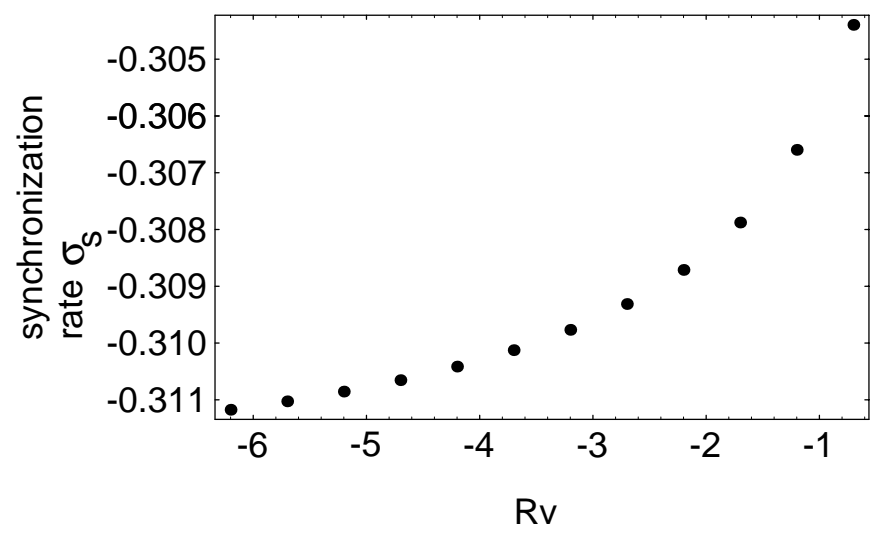

Fig. 10. Synchronization rate $\sigma_{\mathrm{s}}$ for the piecewise linear model, for a $v$-only coupling with a linear dependency on postsynaptic potential, plotted against coupling strength $R_{v} .\left(\Delta=200, T=190, \Delta t=64, E_{v}=0.1, V_{\mathrm{rev}}=-1.7, b\right.$ and $c$ are the same as in Fig. 3).

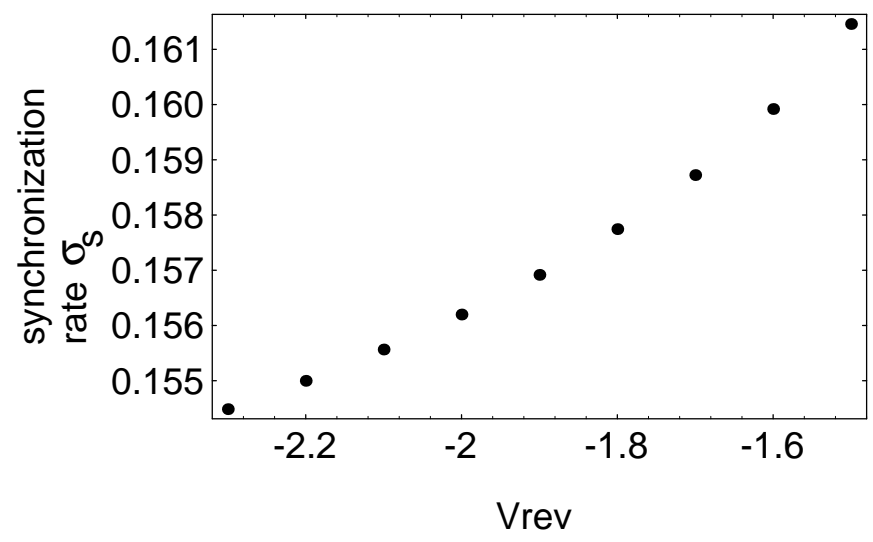

Fig. 11. Synchronization rate $\sigma_{\mathrm{S}}$ for the piecewise linear model, for a $v$-only coupling with a linear dependency on postsynaptic potential, plotted against reversal potential $V_{\text {rev }}\left(\Delta=200, T=190, \Delta t=64, E_{v}=0.1, R_{v}=-2.0, b\right.$ and $c$ are the same as in Fig. 3).

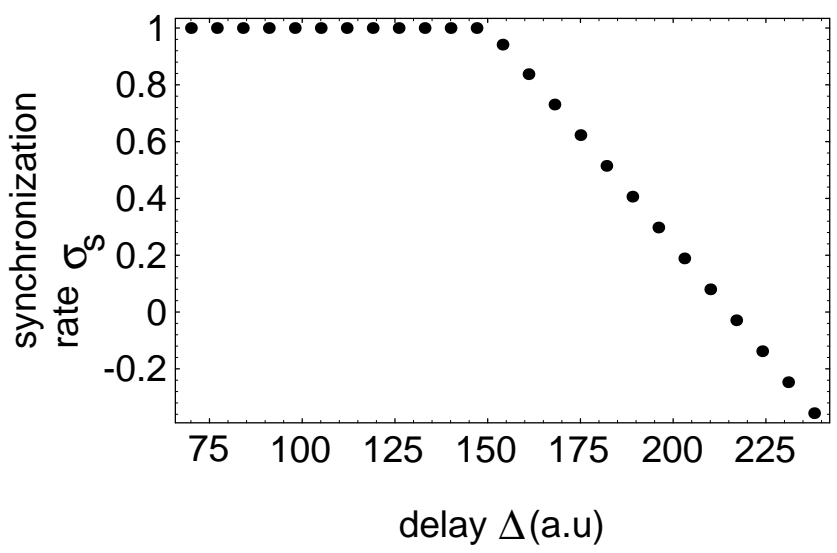

Fig. 12. Synchronization rate $\sigma_{\mathrm{s}}$ for the piecewise linear model, for a $v$-only coupling with a linear dependency on postsynaptic potential, plotted against coupling delay $\Delta\left(T=185, \Delta t=64, E_{v}=0.1, R_{v}=-2.0, V_{\mathrm{rev}}=-2.3, b\right.$ and $c$ are the same as in Fig. 3). 


\section{A system of two units: A more complete inhibitory-type coupling}

\subsection{Description of the new coupling and its neurophysiological justification}

Synapses on neuronal cell bodies are often inhibitory [5,20] which maximizes their effect [2]. Inhibition may result at least in two processes [16,21]. Firstly, an IPSP can hyperpolarize the membrane potential and can move it further away from threshold. Secondly, the inhibition induces an increase of the membrane conductance through the increase of the membrane permeability to $\mathrm{Cl}^{-}$(or $\mathrm{K}^{+}$), which stabilizes the membrane potential near $E_{\mathrm{Cl}^{-}}($or $E_{\mathrm{K}^{+}}$), reducing the amplitude of concomitant EPSPs.

Let us assume that a model neuron can emit action potentials with only two kinds of ionic channels such as $\mathrm{Na}^{+}$and $\mathrm{K}^{+}$in the Hodgkin-Huxley model [19], or $\mathrm{Ca}^{2+}$ and $\mathrm{K}^{+}$in the model of Morris and Lecar [26]. We wish to take into account at least qualitatively the membrane conductance rise due to $\mathrm{K}^{+}$during inhibition. We first recall some observations on the timescales of ionic channel dynamics in the above two models. In the model of Morris and Lecar, $\mathrm{K}^{+}$channel dynamics are much slower than those of $\mathrm{Ca}^{2+}$. It is this property that allows to obtain from the full system of three coupled nonlinear equations the well-known reduced system where the $\mathrm{Ca}^{2+}$ conductance is taken equal to its steady state value, depending only on the potential. In the HodgkinHuxley model, dimensional reduction schemes [1,22] are based, among other things, on the fact that the timescales of the ionic channels for $\mathrm{Na}^{+}$and $\mathrm{K}^{+}$are quite different. Thus instead of modifying the membrane conductance associated with ionic channels to ensure hyperpolarization in the current equation (i.e., the $v$-equation) by means of an instantaneous change of this membrane conductance we shall modify this dynamical equation. This is the $\dot{N}$ equation in the model of Morris and Lecar and the $\dot{n}$ equation in the Hodgkin-Huxley model. In order not to add a further equation to our model (although this can be done), and to keep the usefulness of plane analysis, we keep the usual $I_{v}$ and we just add a constant positive term $I_{u}$ on the RHS of Eq. (2). Thus, we obtain

$$
\begin{aligned}
& \dot{v}=-v^{3}+3 v+2-u+I_{v}+E_{v}, \\
& \dot{u}=c\left(\gamma(1+\tanh (v / \beta))-b u+I_{u}\right)
\end{aligned}
$$

Since $u$ is the recovery variable, a $u$-increase corresponds to an increase of the membrane conductances linked to hyperpolarizing ionic channels or to a decrease of membrane conductances linked to depolarizing ionic channels. A description of this effect can be found for the Hodgkin-Huxley model in [4, Appendix]: "an increase in $n$ corresponds to an increase in the fraction of open potassium channels, and hence an increase in outward current", and in the same way, an increase in $1-h$ corresponds also to an increase of outward current. Therefore, the new positive term $I_{u}$ will mimic the increase of the membrane conductances linked to hyperpolarizing channels during the inhibition duration ( $I_{v}$ and $I_{u}$ are different from zero only during inhibition period, see Fig. 13). For instance, if we assume that the inhibition, in our neuron model, is mediated through ligand-gated channels like the inhibitory $\mathrm{GABA}_{A}[16]$, then we shall consider that the $u$-increase due to the coupling term $I_{u}$ is linked to an increase of the fraction of open ligand-gated channels.

Our coupling model acting on both $v$ and $u$ variables is only an approximation firstly because our neuron model is described by only two equations, and secondly because the hyperpolarizing conductance rise will last as long as the inhibition duration. This may be true for inhibition durations less than or equal to the hyperpolarizing channels' time constant, but it would be incorrect for longer inhibition. In that case, we should describe inhibition at the hyperpolarizing channels level with a more complex dynamics like the kinetic model description [10]. 


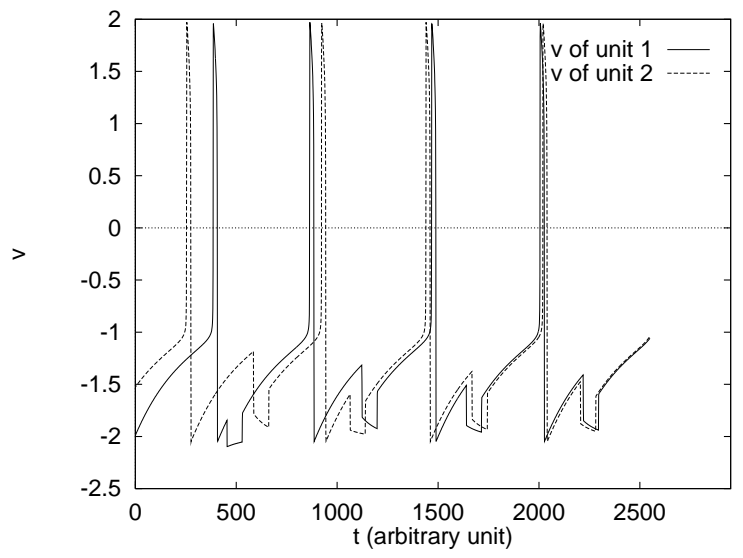

(a) $v$ versus time

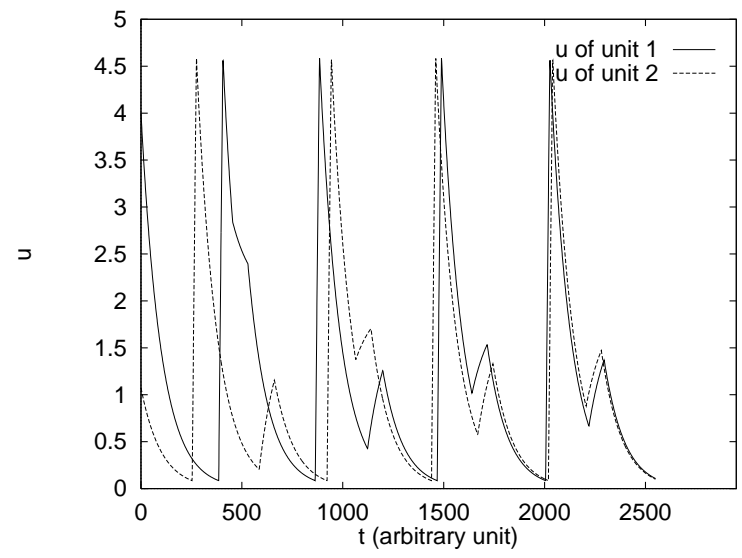

(b) $u$ versus time

Fig. 13. Example of an impulse synchronization, through order reversal, in two units mutually coupled with inbibitory delayed coupling in $v$ and $u\left(E_{v}=0.1, I_{v}=-2.2, I_{u}=0.5, T=75, \Delta=200, b, c, \gamma\right.$, and $\beta$ are the same as in Fig. 3).

\subsection{The synchronization mechanism}

With our more complete coupling, synchronization is reached by order reversal (for most parameters values) (see Fig. 13). The coupling delays the spiking and, as it is required by impulse synchronization, the absolute time between the firing of the two units is reduced.

In the phase plane, with this coupling, we observe that the $u$-nullcline is now translated upward (it will be denoted by $\mathcal{U}_{\text {inhib }}$ ) and the $v$-nullcline is translated downward (as in the previous section it will be denoted by $\mathcal{V}_{\text {inhib }}$ ). Thus, with this coupling, the rest point $S$ is translated upward in $u$ compared to $S^{\prime}$ which is (see above) the rest point with the $v$-only coupling. Therefore, the situation is more complicated than with $v$-only coupling because $S$ is no longer below all the possible points reachable by the transient trajectories between $\mathcal{V}_{\text {free }}$ and $\mathcal{V}_{\text {inhib }}$ (see Fig. 2).

As we again work in the singular limit solutions, we neglect the transient periods where the units move from one set of nullclines to another. From the moment that $I_{u}$ value is sufficient to translate $S$ above the knee LK of the "free" system we have two kinds of possible trajectories in the phase plane for a postsynaptic unit receiving inhibition.

(i) The unit will reach $\mathcal{L}_{\text {inhib }}$ above $S$.

(a) It follows $\mathcal{L}_{\text {inhib }}$ downward without reaching $S$ during inhibition duration. At the end of the inhibition $v$ nearly recovers the value it would have without inhibition. Thus, in this case, the induced delay is weak compared to the inhibition period.

(b) It follows $\mathcal{L}_{\text {inhib }}$ downward and reaches $S$ before the inhibition ends. In this case, the induced delay is equal to $T$ minus the time spent to reach $S$.

(ii) The unit will reach $\mathcal{L}_{\text {inhib }}$ below $S$.

(a) It follows $\mathcal{L}_{\text {inhib }}$ upward without reaching $S$ during inhibition duration. When the inhibition is released, the unit will rejoin $\mathcal{L}_{\text {free }}$. Thus the induced delay, in comparison with a "free" unit, is equal to $T$ plus the time needed by the unit to rejoin its initial position in $\mathcal{L}_{\text {free }}$. Therefore, the delay is greater than the inhibition duration.

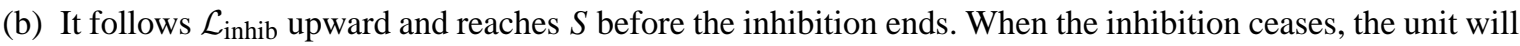
rejoin $\mathcal{L}_{\text {free }}$. Thus, the delay, in comparison with a "free" unit, is equal to $T$ minus the time spent to reach $S$, plus the time needed to return to its initial position. 


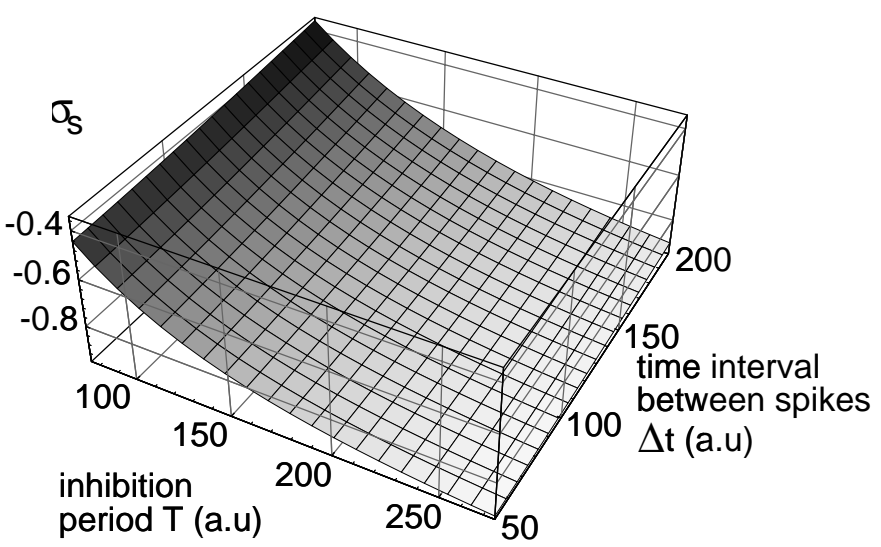

Fig. 14. Synchronization rate $\sigma_{\mathrm{s}}$ for the piecewise linear model plotted versus inhibition period and time interval between the spikes of the two units ( $\Delta=200, E_{v}=0.1, I_{u}=0.4, b$ and $c$ are the same as in Fig. 13).

For these different cases, the effect of inhibition on the postsynaptic unit strongly depends on its position in the phase space and ensures that the more advanced unit will be more delayed than the lagged one. We shall compute the magnitude of this effect in the piecewise linear model.

\subsection{Piecewise linear model}

We shall use the piecewise linear model with the new coupling term which acts only during the duration of the inhibition. Then, one gets

$$
\mathbf{a}=\left\{\begin{array}{cl}
\mathbf{a}_{i}=\left(\begin{array}{c}
c I_{u} \\
-k V_{Q}+l+I_{v}+E_{v}
\end{array}\right) & \text { if the system is "inhibited", } \\
0 \\
\mathbf{a}_{\mathrm{f}}=\left(\begin{array}{c}
0 \\
-k V_{Q}+l+E_{v}
\end{array}\right)
\end{array} \quad\right. \text { if the system is "free". }
$$

In order to ensure that the fixed point $S$ is such that $V_{\mathrm{s}}<V_{\text {th }}$ we shall only consider the case where $-I_{v}+I_{u} / b>E_{v}$, i.e., $-I_{v}>E_{v}$ given that $I_{u}>0$. The detailed calculations are done in Appendix B.1. One gets

$$
\sigma_{\mathrm{s}}=\frac{1}{\lambda_{1} \Delta t} \ln \left(\frac{J+u_{0} \exp \left(\lambda_{1}(\Delta t+T+\Delta)\right)}{J+u_{0} \exp \left(\lambda_{1}(-\Delta t+T+\Delta)\right)}\right)-1
$$

where $J=\left(I_{u} / b\right)\left(1-\exp \left(\lambda_{1} T\right)\right)$.

Thus we obtain an analytical solution for the synchronization rate, $\sigma_{\mathrm{s}}$, which is seen to depend only on $I_{u}$ and not on $I_{v}$. The dependence on $I_{v}$ has been removed on the RHS of Eq. (B.6) consequently to the approximations used in deriving Eqs. (B.3) and (B.4). We can see that the effect of synchronization is reduced for a longer period of inhibition and a longer coupling delay (see Fig. 14). The dependency of $\sigma_{\mathrm{s}}$ on the coupling strength is shown in Fig. 15.

The results are in very good agreement (see Fig. 16) with the numerical simulations of the full system defined by Eqs. (12) and (13). The agreement is better than 3.5\%. We note that the absolute value of the synchronization rate is smaller in the full system than in the piecewise linear one, which corresponds to a faster synchronization. This holds for all the initial conditions which we have tried. Heuristically, this can be understood because the postsynaptic position dependency is stronger in the full system than in the piecewise linear one, thus improving the 


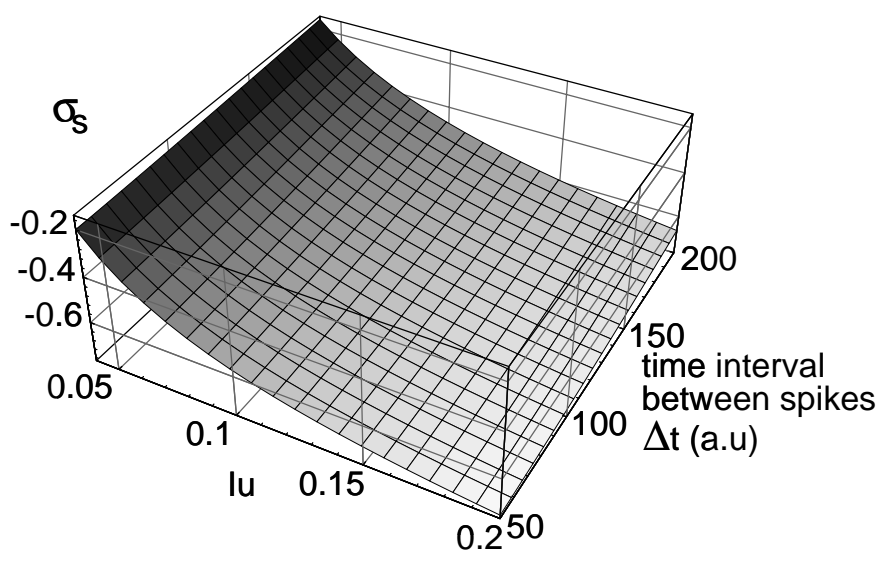

Fig. 15. Synchronization rate $\sigma_{\mathrm{s}}$ for the piecewise linear model versus the coupling strength $I_{u}$ and the spike time intervals $\Delta t$ of the two units ( $\Delta=200, T=200, E_{v}=0.10, b$ and $c$ are the same as in Fig. 13).

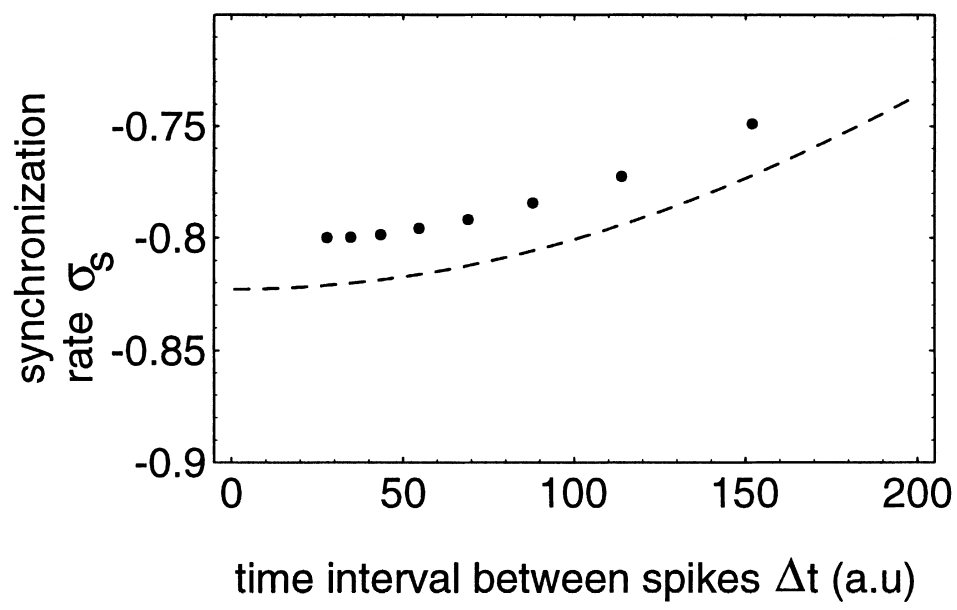

Fig. 16. Comparison between the synchronization rate, $\sigma_{\mathrm{s}}$, obtained from the numerical results (dots) of two neurons, with $u-v$ inhibitory-type coupling, with the one obtained from analytical results (dashed line) with the piecewise linear model. The minus sign corresponds to a reversal in the order of spiking times $\left(T=150, \Delta=200, I_{u}=0.5, I_{v}=-2.0, E_{v}=0.1, b\right.$ and $c$ are the same as in Fig. 13).

synchronization. The effects of the nonlinear dependency on $v$ of the RHS of Eq. (12) are particularly important when the time interval between the spikes of the two units is small compared to the repolarization period.

The accuracy of the analytical results is better in this section than in Section 3. This might result from the existence, in the $v$-only coupling, of two relevant timescales in the nonlinear system (see Eq. (A.15)) whereas it is not the case with the more complete coupling acting in both $u$ and $v$.

We can also calculate the synchronization rate when one considers coupling terms with a linear dependency on postsynaptic potential (the detailed calculus is done in Appendix B.2). The results are shown in Figs. 17-19. The behaviour of $\sigma_{\mathrm{s}}$ as a function of the time interval between the spikes of the two units is similar to the one obtained from the constant $u-v$ coupling. The synchronization rate as a function of the coupling delay $\Delta$ is shown in Fig. 20. Its behaviour is qualitatively the same for the constant $u-v$ coupling. It is worth noting that, for a fixed value of the delay, the synchronization rate can be made small by means of a suitable choice of other coupling parameters. 


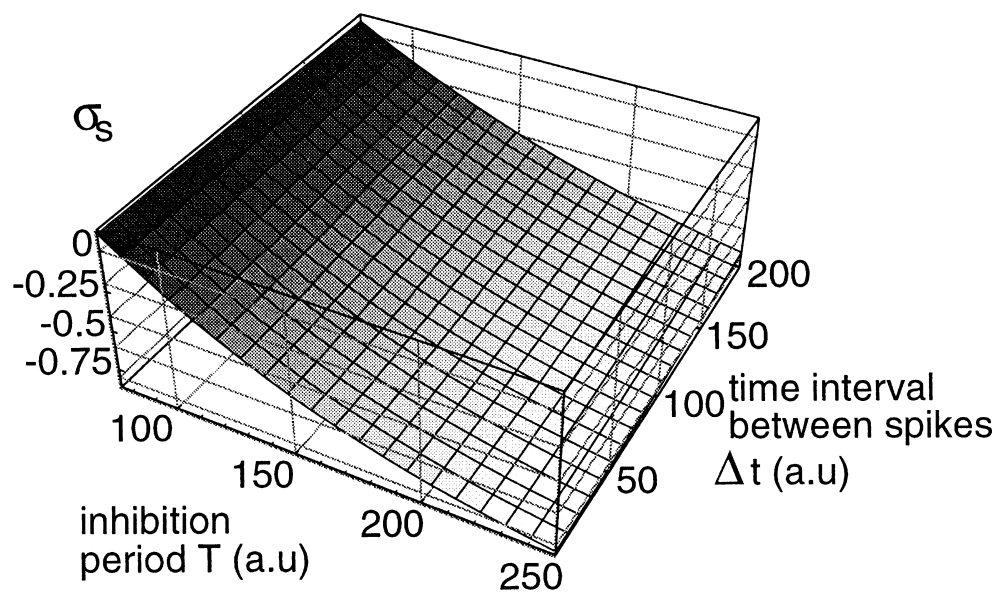

Fig. 17. Synchronization rate $\sigma_{\mathrm{S}}$ for the piecewise linear model, for a $u-v$ coupling with a linear dependency on postsynaptic potential, plotted against inhibition period $T$ and time interval $\Delta t$ between the spikes of the two units $\left(\Delta=200, E_{v}=0.1, R_{u}=0.4, R_{v}=-2.5\right.$, $V_{s}=-1.75, b$ and $c$ are the same as in Fig. 13).

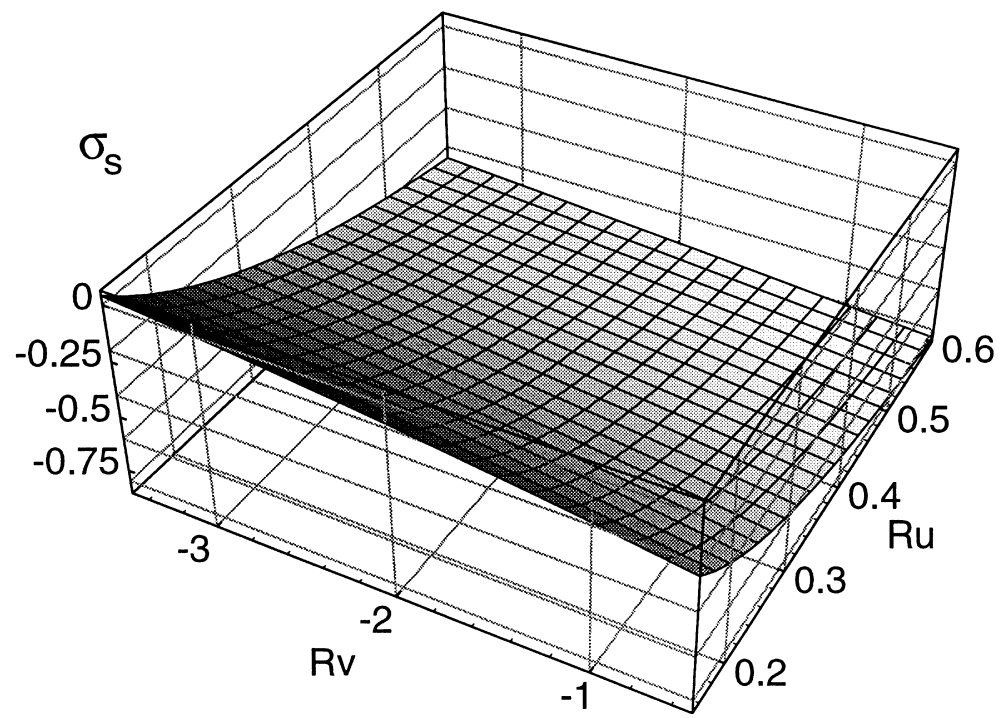

Fig. 18. Synchronization rate $\sigma_{\mathrm{s}}$ for the piecewise linear model, for a $u-v$ coupling with a linear dependency on postsynaptic potential, plotted against coupling strength on $v$-variable $R_{v}$ and coupling strength on $u$-variable $R_{u}\left(\Delta=150, T=200, \Delta t=75, E_{v}=0.1\right.$, $V_{\text {rev }}=-1.75, b$ and $c$ are the same as in Fig. 13).

\section{Summary and discussion}

We have shown that neural oscillators can rapidly reach impulse synchronization through a time-delayed coupling, with non-vanishing duration, by considering a more realistic inhibitory coupling than the one acting only on variable $v$. With this $v$-coupling, we have shown that impulse synchronization can be reached only for a small particular interval of values of the duration of the inhibition. Besides, the position of this interval depends on the values of the external stimulation. This coupling only describes current injection in the soma associated with inhibition whereas 


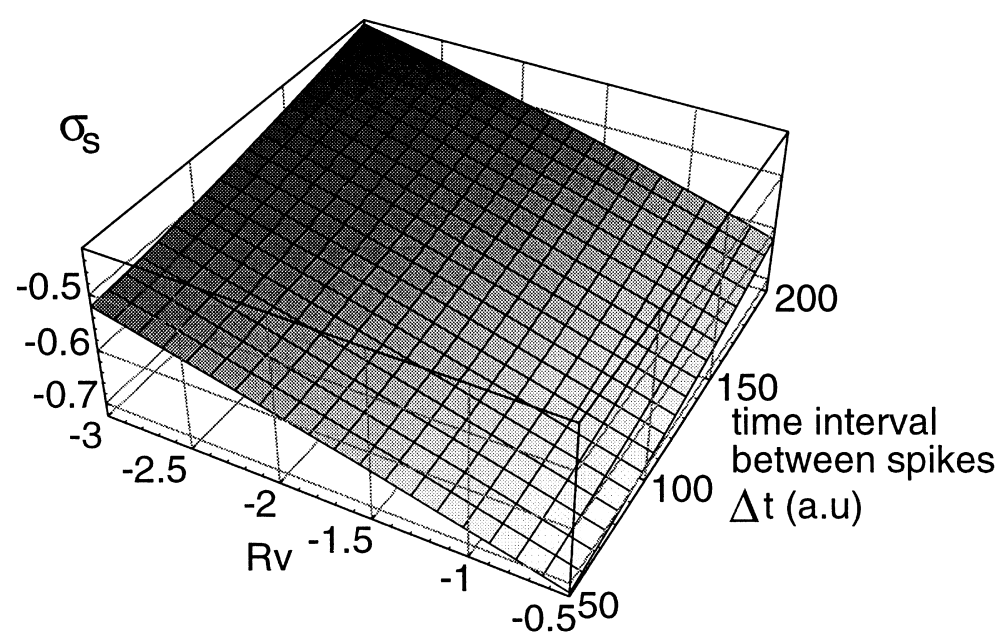

Fig. 19. Synchronization rate $\sigma_{\mathrm{s}}$ for the piecewise linear model, for a $u-v$ coupling with a linear dependency on postsynaptic potential, plotted against coupling strength on $v$-variable $R_{v}$ and reversal potential $V_{\text {rev }}\left(\Delta=150, T=200, \Delta t=75, E_{v}=0.1, R_{u}=0.4, b\right.$ and $c$ are the same as in Fig. 13).

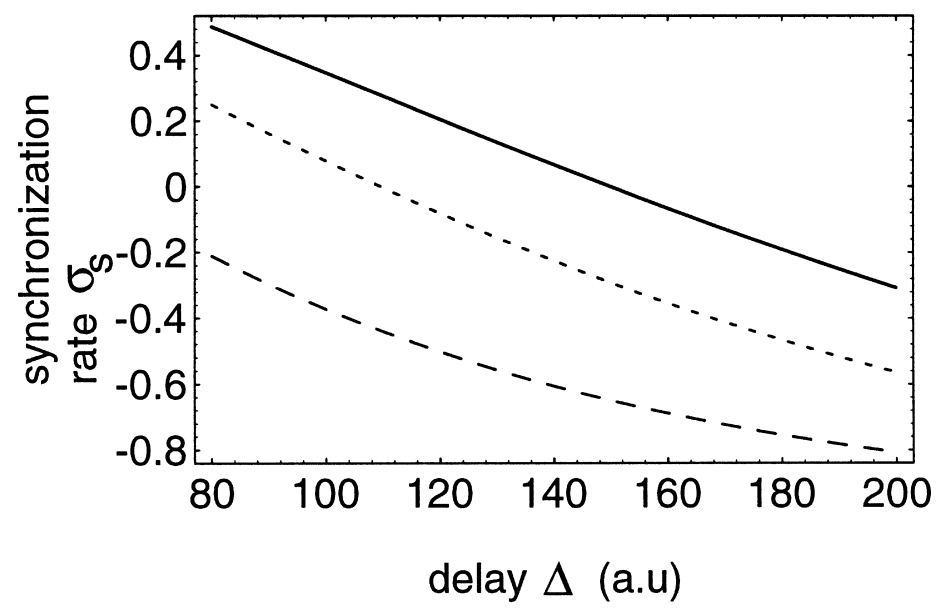

Fig. 20. Synchronization rate $\sigma_{\mathrm{S}}$ for the piecewise linear model, for a $u-v$ coupling with a linear dependency on postsynaptic potential, plotted against time delay $\Delta$ for three sets of parameters: $R_{u}=0.2, R_{v}=-2.0, V_{\mathrm{rev}}=-1.6$ (solid line), $R_{u}=0.4, R_{v}=-3.0$, $V_{\mathrm{rev}}=-1.6$ (dotted line), and $R_{u}=0.4, R_{v}=-3.0, V_{\mathrm{rev}}=-2.2$ (dashed line) (For both curves $T=200, \Delta t=75, E_{v}=0.1, b$ and $c$ are the same as in Fig. 13).

the $u-v$ coupling we propose takes also into account the increase in membrane conductance produced by inhibition. We analyse this approximately by means of a phase-plane analysis. A more accurate study of this effect is possible if one works with a true conductance-based model neuron and not with a two-dimensional model; the building of a piecewise linear model allows however analytical insights. In the case of the $u-v$ coupling, we give an analytical expression for the synchronization rate. For the $v$-only coupling, we give only local analytical approximations of the synchronization rate (see Appendix A.1) but we obtain a global numerical solution. Our approach may be extended to other two-component neuronal models, if two different timescales are present, and if the nullclines may be, at least, approximately piecewise linearized. 
For both inhibitory couplings, the trajectories of each unit in the two-dimensional phase-plane are strongly modified, during the inhibition, compared to the trajectories of a "free" unit. When one of the two units reaches a stable fixed point, we cannot consider that the coupling has induced only a perturbation of the limit-cycle of the uninhibited system, especially when the unit goes upward in the phase plane, along $\mathcal{L}_{\text {inhib }}$, towards $S$, where it remains until the inhibition is removed. This remark agrees with that of Somers and Kopell $[36,37]$ about the different behaviour of phase-type two-component neuron models compared with the same models in a relaxation-type regime.

To study the dependence of the synchronization on the coupling strength, we must distinguish between the two kinds of inhibition. For the $v$-only inhibition, the synchronization rate does not depend on $I_{v}$ or depends only slightly on it according to the detailed cases described in Section 3.1. In the piecewise linear model with a constant coupling, this can easily be understood if we remember firstly that the stable fixed point $S^{\prime}$ is below all the possible points reachable by the transient trajectories between the $v$-nullcline of the "free" system and the one of the inhibited system, and, secondly that the slope $k$ of the linearized $v$-nullcline is constant whatever the coupling strength. When the coupling term depends linearly on postsynaptic potential the slope of the linearized $v$-nullcline is weakly changed during inhibition duration. Consequently, the synchronization rate dependency on the coupling strength is weakly increased. On the other hand, with the inhibition acting both on $u$ and $v$, Eq. (15) leads to a synchronization rate which strongly depends on the coupling strength (see Fig. 15). This result can be easily understood, since in this case the position of the stable fixed point $S$ strongly depends on $I_{u}$.

For the $v$-only coupling, the results on synchronization are quite different from the ones obtained for the leaky integrate-and-fire model (LIF) where the coincidence synchronization can be reached from a wider range of initial conditions and durations of inhibition [28]. This discrepancy comes form the fact that in the LIF model, time dependent conductances are neglected whereas they are included in the two-component neuronal model used herein.

At the end of their paper [36], Somers and Kopell note that "if the coupling has another form (e.g., electrical) or delays are significant, the conclusions of the analysis and the simulations may not hold". One has to remind this warning when one compares their results and the results of Terman and Wang with those obtained in our study. With the two-component dynamical neuron model we use, instantaneous excitatory coupling lead to synchronization of two mutually coupled oscillators [36, Theorem] and the role of the global inhibition introduced by Terman and Wang [38] is to desynchronize two oscillators without direct excitatory coupling whereas our delayed inhibitory coupling leads to synchronization. It is worth noting that the synchronization mechanisms acting herein are different from the fast threshold modulation (FTM) first described in [36] for an instantaneous excitatory coupling since the ratio of the rates of change of $u$ before and after the jump from $\mathcal{L}_{\text {free }}$ to $\mathcal{R}_{\text {free }}$ does not play any part whereas it is a "key notion" in FTM. Herein the key point is the existence and the position of the rest points. With the couplings we use, we have not to consider the right branches of the $v$-nullclines to study synchronization. Therefore, as already mentionned in Section 3, no amplitude effect induced by the coupling is present on the spiking process whereas the FTM mechanism leads to a "solution with lower frequency and higher amplitude" [36]. In [36, Fig. 4], a piecewise linear approximation of the $v$-nullclines is sketched to show that, in this case, the FTM does not guarantee synchrony, on the contrary we have shown that synchrony can be reached in a piecewise linear approximation of the two-component dynamical neuron model used herein if we consider the delayed inhibitory coupling described in Section 4. Consequently, it is not possible to use the piecewise linear approximation to get an explicit analytical formula for the synchronization rate in the case of the FTM.

In the present paper, inhibition duration is one of the key parameters of synchronization through mutual delayed inhibition. This result agrees with the experimental results obtained by Whittington and his colleagues [41]. They show that the frequency of the inhibitory neuronal network oscillations is a function of IPSP kinetics in individual inhibitory interneurons. Inhibition duration is often neglected in other studies, either because it vanishes, as in pulse-coupled models [11] or in phase models [35], or because the main parameter is the coupling delay [28]. A functional role can be found in a globally coupled inhibitory network. As has been proposed in [8], networks of 
inhibitory interneurons "can provide a clock signal for the timing of action potentials of cortical principal cells", but in order to do so, the "multistable clusters synchronized in common phases" found by Ernst and his colleagues [11] in a globally pulse-coupled network of inhibitory integrate-and-fire neurons with time-delayed coupling should be avoided. We believe that this is possible, even for small temporal delay (compared to the averaged interspike interval), with the neuronal model and the inhibitory coupling we use herein.

In future work we shall investigate this aspect within the framework of the present model as well as effects of the noise in the delays and in the neuronal dynamics. Furthermore, we shall investigate another functional role in rapid pattern segmentation [38] through the temporal labelling of each stimulus within a locally connected two-dimensional network. Preliminary results are described in [32].

\section{Acknowledgements}

It is a pleasure to thank O. Parodi, D. Hansel, C. Meunier and H.C. Tuckwell for a careful reading of this work and for their comments and suggestions.

\section{Appendix A. Calculation of the synchronization rate in the piecewise linear model for a coupling on the $v$ component}

\section{A.1. Constant coupling}

We start from Eq. (8). Since det $(\mathbf{M})=-b c k$ and $\operatorname{Tr}(\mathbf{M})=k-b c$, one can compute $\mathbf{M}^{-1}$ and diagonalize $\mathbf{M}$ by means of the transformation $\mathbf{D}=\mathbf{C}^{-1} \mathbf{M C}$, where $\mathbf{D}$ is equal to diag $\left(\begin{array}{ll}\lambda_{1} & \lambda_{2}\end{array}\right)$. Keeping only leading order terms, with $\lambda_{1}=-b c$ and $\lambda_{2}=k$, we obtain

$$
\begin{aligned}
\mathbf{M}^{-1} & =\frac{1}{\lambda_{1} \lambda_{2}}\left(\begin{array}{cc}
\lambda_{2} & 0 \\
1 & \lambda_{1}
\end{array}\right), \\
\mathbf{C} & =\left(\begin{array}{cc}
1 & 0 \\
\left(\lambda_{2}-\lambda_{1}\right)^{-1} & 1
\end{array}\right), \\
\mathbf{C}^{-1} & =\left(\begin{array}{cc}
1 & 0 \\
-\left(\lambda_{2}-\lambda_{1}\right)^{-1} & 1
\end{array}\right) .
\end{aligned}
$$

The solution Eq. (7), with initial value $\mathbf{u}(0)=\left(\begin{array}{l}u_{0} \\ v_{0}\end{array}\right)$, is easily found:

$$
\mathbf{u}(t)=-\mathbf{M}^{-1} \mathbf{a}+\mathbf{C} \exp (\mathbf{D} t) \mathbf{C}^{-1}\left(\mathbf{u}(0)+\mathbf{M}^{-1} \mathbf{a}\right) .
$$

Using matrix formalism, with $\mathbf{u}_{1}\left(t_{1}\right)=\mathbf{u}_{2}\left(t_{2}\right)=\mathbf{u}(0)$, we get:

$$
\begin{aligned}
\mathbf{u}_{1}\left(t_{2}+\Delta\right) & =-\mathbf{M}^{-1} \mathbf{a}_{\mathrm{f}}+\mathbf{C} \exp \left(\mathbf{D}\left(t_{2}+\Delta-t_{1}\right)\right) \mathbf{C}^{-1}\left(\mathbf{u}_{1}\left(t_{1}\right)+\mathbf{M}^{-1} \mathbf{a}_{\mathrm{f}}\right) . \\
\mathbf{u}_{2}\left(t_{1}+\Delta\right) & =-\mathbf{M}^{-1} \mathbf{a}_{\mathrm{f}}+\mathbf{C} \exp \left(\mathbf{D}\left(t_{1}+\Delta-t_{2}\right)\right) \mathbf{C}^{-1}\left(\mathbf{u}_{2}\left(t_{2}\right)+\mathbf{M}^{-1} \mathbf{a}_{\mathrm{f}}\right) . \\
\mathbf{u}_{1}\left(t_{2}+\Delta+T\right) & =-\mathbf{M}^{-1} \mathbf{a}_{\mathrm{i}}+\mathbf{C} \exp (\mathbf{D} T) \mathbf{C}^{-1}\left(\mathbf{u}_{1}\left(t_{2}+\Delta\right)+\mathbf{M}^{-1} \mathbf{a}_{\mathbf{i}}\right) . \\
\mathbf{u}_{2}\left(t_{2}+\Delta+T\right) & =-\mathbf{M}^{-1} \mathbf{a}_{\mathrm{i}}+\mathbf{C} \exp (\mathbf{D} T) \mathbf{C}^{-1}\left(\mathbf{u}_{2}\left(t_{1}+\Delta\right)+\mathbf{M}^{-1} \mathbf{a}_{\mathrm{i}}\right) . \\
\mathbf{u}_{1}\left(t_{2}+\Delta+T+\Delta t_{1}\right) & =-\mathbf{M}^{-1} \mathbf{a}_{\mathrm{f}}+\mathbf{C} \exp \left(\mathbf{D}\left(\Delta t_{1}\right)\right) \mathbf{C}^{-1}\left(\mathbf{u}_{1}\left(t_{2}+\Delta+T\right)+\mathbf{M}^{-1} \mathbf{a}_{\mathrm{f}}\right) . \\
\mathbf{u}_{2}\left(t_{1}+\Delta+T+\Delta t_{2}\right) & =-\mathbf{M}^{-1} \mathbf{a}_{\mathrm{f}}+\mathbf{C} \exp \left(\mathbf{D}\left(\Delta t_{2}\right)\right) \mathbf{C}^{-1}\left(\mathbf{u}_{2}\left(t_{1}+\Delta+T\right)+\mathbf{M}^{-1} \mathbf{a}_{\mathrm{f}}\right) .
\end{aligned}
$$


After substitutions and simplifications:

$$
\begin{aligned}
\mathbf{u}_{1}\left(t_{2}+\Delta+T+\Delta t_{1}\right)= & -\mathbf{M}^{-1} \mathbf{a}_{\mathrm{f}}+\mathbf{C} \exp \left(\mathbf{D}\left(\Delta t_{1}\right)\right) \mathbf{C}^{-1} \mathbf{M}^{-1}\left(\mathbf{a}_{\mathrm{f}}-\mathbf{a}_{\mathrm{i}}\right) \\
& +\mathbf{C} \exp \left(\mathbf{D}\left(\Delta t_{1}+T+\Delta+\Delta t\right)\right) \mathbf{C}^{-1}\left(\mathbf{u}(0)+\mathbf{M}^{-1} \mathbf{a}_{\mathrm{f}}\right) \\
& -\mathbf{C} \exp \left(\mathbf{D}\left(\Delta t_{1}+T\right)\right) \mathbf{C}^{-1} \mathbf{M}^{-1}\left(\mathbf{a}_{\mathrm{f}}-\mathbf{a}_{\mathrm{i}}\right) . \\
\mathbf{u}_{2}\left(t_{1}+\Delta+T+\Delta t_{2}\right)= & -\mathbf{M}^{-1} \mathbf{a}_{\mathrm{f}}+\mathbf{C} \exp \left(\mathbf{D}\left(\Delta t_{2}\right)\right) \mathbf{C}^{-1} \mathbf{M}^{-1}\left(\mathbf{a}_{\mathrm{f}}-\mathbf{a}_{\mathrm{i}}\right) \\
& +\mathbf{C} \exp \left(\mathbf{D}\left(\Delta t_{2}+T+\Delta-\Delta t\right)\right) \mathbf{C}^{-1}\left(\mathbf{u}(0)+\mathbf{M}^{-1} \mathbf{a}_{\mathrm{f}}\right) \\
& -\mathbf{C} \exp \left(\mathbf{D}\left(\Delta t_{2}+T\right)\right) \mathbf{C}^{-1} \mathbf{M}^{-1}\left(\mathbf{a}_{\mathrm{f}}-\mathbf{a}_{\mathrm{i}}\right) .
\end{aligned}
$$

(We can easily check that, for $T=0$, we get the expected results.) We now have to evaluate the RHS of Eqs. (A.11) and (A.12). We have from Eq. (9):

$$
\begin{aligned}
\mathbf{M}^{-1} \mathbf{a}_{\mathrm{f}} & =\frac{1}{k}\left(\begin{array}{c}
0 \\
-k V_{Q}+l+E_{v}
\end{array}\right), \\
\mathbf{M}^{-1}\left(\mathbf{a}_{\mathrm{f}}-\mathbf{a}_{\mathrm{i}}\right) & =\left(\begin{array}{c}
0 \\
-I_{v} / k
\end{array}\right) .
\end{aligned}
$$

The first components of the vectors defined by Eqs. (A.13) and (A.14) vanish. Therefore, the terms in $\exp \left(\lambda_{2} t\right)$ must be kept, even if $\exp \left(\lambda_{2} t\right) \ll \exp \left(\lambda_{1} t\right)$ (recall that $\lambda_{1}$ and $\lambda_{2}<0$ and $\left.\lambda_{1} / \lambda_{2}=-b c / k \ll 1\right)$. Thus, we get

$$
\mathbf{C} \exp (\mathbf{D} t) \mathbf{C}^{-1}=\left(\begin{array}{cc}
\exp \left(\lambda_{1} t\right) & 0 \\
\left(\exp \left(\lambda_{1} t\right)-\exp \left(\lambda_{2} t\right)\right) /\left(\lambda_{2}-\lambda_{1}\right) & \exp \left(\lambda_{2} t\right)
\end{array}\right) .
$$

We can compute $\Delta t_{1}$ from Eq. (A.11) using the threshold condition on $v$ for unit 1, i.e., $v_{1}\left(t_{2}+\Delta+T+\Delta t_{1}\right)=V_{\text {th }}$. Since $k\left(V_{\mathrm{th}}-V_{Q}\right)+l=0$, we get

$$
\begin{aligned}
E_{v}= & -I_{v} \exp \left(\lambda_{2} \Delta t_{1}\right)\left(1-\exp \left(\lambda_{2} T\right)\right)-\left(\frac{\lambda_{1}}{\lambda_{2}-\lambda_{1}}\right) u_{0} \exp \left(\lambda_{2}\left(\Delta t_{1}+T+\Delta+\Delta t\right)\right) \\
& +\left(\frac{\lambda_{2}}{\lambda_{2}-\lambda_{1}}\right) u_{0} \exp \left(\lambda_{1}\left(\Delta t_{1}+T+\Delta+\Delta t\right)\right)
\end{aligned}
$$

Using a similar equation for unit 2 , we can compute $\Delta t_{2}$ from Eq. (A.12):

$$
\begin{aligned}
E_{v}= & -I_{v} \exp \left(\lambda_{2} \Delta t_{2}\right)\left(1-\exp \left(\lambda_{2} T\right)\right)-\left(\frac{\lambda_{1}}{\lambda_{2}-\lambda_{1}}\right) u_{0} \exp \left(\lambda_{2}\left(\Delta t_{2}+T+\Delta-\Delta t\right)\right) \\
& +\left(\frac{\lambda_{2}}{\lambda_{2}-\lambda_{1}}\right) u_{0} \exp \left(\lambda_{1}\left(\Delta t_{2}+T+\Delta-\Delta t\right)\right) .
\end{aligned}
$$

These two equations can be solved numerically, the results being shown in Figs. 5 and 6 .

With the above formula, $\sigma_{\mathrm{s}}$ can be computed only numerically. Nevertheless, for the three cases defined in the last part of Section 3.1, we can approximate $\Delta t_{1}$ and $\Delta t_{2}$ in each case, and consequently we get analytical results for $\sigma_{\mathrm{s}}$.

- Case (i): Since both $\Delta t_{1}$ and $\Delta t_{2}$ differ from zero, we can neglect the exponential terms in $\lambda_{2}$ compared to the ones in $\lambda_{1}$ in Eqs. (A.16) and (A.17). Thus, $\Delta t_{2}-\Delta t_{1}=2 \Delta t$, and then from Eq. (11), $\sigma_{\mathrm{s}}=1$. Thus $\sigma_{\mathrm{s}}$ does not depend on the coupling strength $I_{v}$.

- Case (ii): Since $\Delta t_{1}$ is nearly equal to zero, one can approximate it, using Eq. (A.16), by

$$
\Delta t_{1}=\frac{1}{\lambda_{2}} \ln \left(\frac{E_{v}-\left(\lambda_{2} /\left(\lambda_{2}-\lambda_{1}\right)\right) u_{0} \exp \left(\lambda_{1}(T+\Delta+\Delta t)\right)}{-I_{v}\left(1-\exp \left(\lambda_{2} T\right)\right)}\right) .
$$




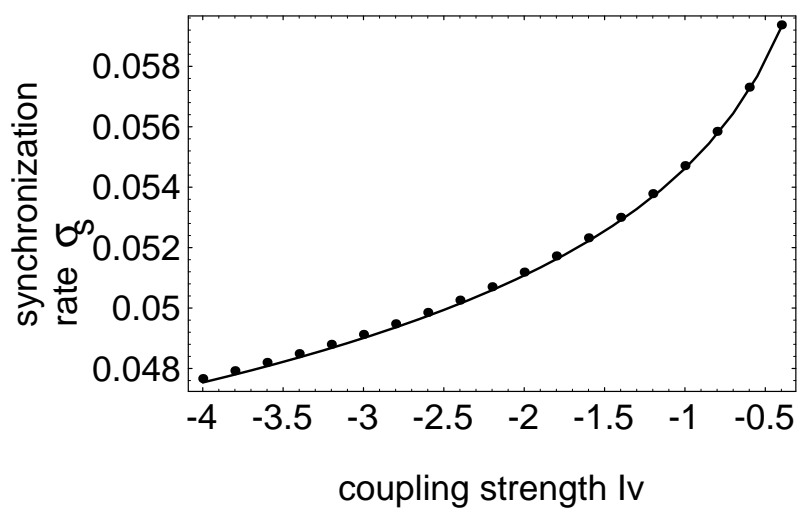

Fig. 21. Comparison between the synchronization rates obtained, in case (ii), for the piecewise linear model, from the numerical solutions (dots) with the one obtained from the approximated analytical results (solid line) $\left(T=150, \Delta=249, E_{v}=0.1, \Delta t=25, b\right.$ and $c$ are the same as in Fig. 3).

Using Eq. (A.17), one finds

$$
\Delta t_{2}=\frac{1}{\lambda_{1}} \ln \left(\frac{E_{v}}{\left(\lambda_{2} /\left(\lambda_{2}-\lambda_{1}\right)\right) u_{0}}\right)-T-\Delta+\Delta t .
$$

Eqs. (A.18), (A.19), and (11) lead to an analytical approximation for $\sigma_{\mathrm{s}}$ which is plotted, together with the numerical solutions of Eqs. (A.16), (A.17), and (11) in Fig. 21. One clearly sees that the approximations we have used are fully justified.

- Case (iii): Since both $\Delta t_{1}$ and $\Delta t_{2}$ are nearly equal to zero, one gets $\Delta t_{2}-\Delta t_{1}=0$ and consequently $\sigma_{\mathrm{s}}=-1$. As in case (i), we see that the synchronization rate does not depend on the coupling strength.

It is worth noting that one cannot use Eq. (A.16) to analyse the case $\Delta t_{1}=0$ straight because this equation is the threshold condition on variable $v$ and inhibitory coupling strength is such as $V_{s^{\prime}}<V_{\text {th }}$. The stable fixed point of Eqs. (5) and (6) can only be reached asymptotically. For the limit case where the first unit reaches the neighbourhood of the stable fixed point we approximately have $P=T+\Delta t+\Delta$ where $P$ is the interspike interval of a free stimulated unit. Therefore, we can calculate a $\Delta_{\text {limit }}$ for fixed $P, \Delta t$, and $T$; this value corresponds to the change of the slope of $\sigma_{\mathrm{s}}$ in Fig. 12.

\section{A.2. Coupling with a linear dependency on postsynaptic potential}

During inhibition, we get the following system:

$$
\begin{aligned}
& \dot{v}=k\left(v-V_{Q}\right)+l-u+\left(v-V_{\mathrm{rev}}\right) R_{v}+E_{v}, \\
& \dot{u}=-c b u .
\end{aligned}
$$

In order to ensure that the firing does not occur during inhibition, the following inequality must hold: $V_{\text {rev }}<$ $V_{\mathrm{th}}+E_{v} / R_{v}$. Since the formalism is the same as in the previous part of this appendix, we just give the main results. We define $\mathbf{M}_{i}$ and its derived matrices $\mathbf{M}_{i}^{-1}, \mathbf{C}_{i}, \mathbf{C}_{i}^{-1}$, and $\mathbf{D}_{i}$. Since these matrices are associated with Eqs. (A.20) and (A.21) one has to consider them only during inhibition duration, we obtain

$$
\frac{\mathrm{d} \mathbf{u}(t)}{\mathrm{d} t}=\mathbf{M}_{i} \mathbf{u}(t)+\mathbf{a}_{i},
$$


where

$$
\begin{aligned}
\mathbf{M}_{i} & =\left(\begin{array}{cc}
-b c & 0 \\
-1 & k+R_{v}
\end{array}\right) \\
\mathbf{a}_{i} & =\left(\begin{array}{c}
0 \\
-k V_{Q}+l-R_{v} V_{\mathrm{rev}}+E_{v}
\end{array}\right) .
\end{aligned}
$$

Keeping only leading order terms, we obtain for the two eigenvalues $\lambda_{i 1}$ and $\lambda_{i 2}$ of $\mathbf{M}_{i}$ :

$$
\lambda_{i 1}=-b c=\lambda_{1} \quad \text { and } \quad \lambda_{i 2}=k+R_{v}=\lambda_{2}+R_{v}
$$

These eigenvalues are used to compute the derived matrices from Eqs. (A.1), (A.2) and (A.3). The new formula corresponding to Eqs. (A.11) and (A.12) are:

$$
\begin{aligned}
\mathbf{u}_{1}\left(t_{2}\right. & \left.+\Delta+T+\Delta t_{1}\right) \\
= & -\mathbf{M}^{-1} \mathbf{a}_{\mathrm{f}}+\mathbf{C} \exp \left(\mathbf{D}\left(\Delta t_{1}\right)\right) \mathbf{C}^{-1}\left(\mathbf{M}^{-1} \mathbf{a}_{\mathrm{f}}-\mathbf{M}_{i}^{-1} \mathbf{a}_{i}\right) \\
& +\mathbf{C} \exp \left(\mathbf{D}\left(\Delta t_{1}\right)\right) \mathbf{C}^{-1} \mathbf{C}_{i} \exp \left(\mathbf{D}_{i}(T)\right) \mathbf{C}_{i}^{-1} \mathbf{C} \exp (\mathbf{D}(\Delta+\Delta t)) \mathbf{C}^{-1}\left(\mathbf{u}(0)+\mathbf{M}^{-1} \mathbf{a}_{\mathrm{f}}\right) \\
& -\mathbf{C} \exp \left(\mathbf{D}\left(\Delta t_{1}\right)\right) \mathbf{C}^{-1} \mathbf{C}_{i} \exp \left(\mathbf{D}_{i}(T)\right) \mathbf{C}_{i}^{-1}\left(\mathbf{M}^{-1} \mathbf{a}_{\mathrm{f}}-\mathbf{M}_{i}^{-1} \mathbf{a}_{i}\right) . \\
\mathbf{u}_{2}\left(t_{1}+\Delta+T+\Delta t_{2}\right) & +\mathbf{M}^{-1} \mathbf{a}_{\mathrm{f}}+\mathbf{C} \exp \left(\mathbf{D}\left(\Delta t_{2}\right)\right) \mathbf{C}^{-1}\left(\mathbf{M}^{-1} \mathbf{a}_{\mathrm{f}}-\mathbf{M}_{i}^{-1} \mathbf{a}_{i}\right) \\
= & +\mathbf{C} \exp \left(\mathbf{D}\left(\Delta t_{2}\right)\right) \mathbf{C}^{-1} \mathbf{C}_{i} \exp \left(\mathbf{D}_{i}(T)\right) \mathbf{C}_{i}^{-1} \mathbf{C} \exp (\mathbf{D}(\Delta-\Delta t)) \mathbf{C}^{-1}\left(\mathbf{u}(0)+\mathbf{M}^{-1} \mathbf{a}_{\mathrm{f}}\right) \\
& -\mathbf{C} \exp \left(\mathbf{D}\left(\Delta t_{2}\right)\right) \mathbf{C}^{-1} \mathbf{C}_{i} \exp \left(\mathbf{D}_{i}(T)\right) \mathbf{C}_{i}^{-1}\left(\mathbf{M}^{-1} \mathbf{a}_{\mathrm{f}}-\mathbf{M}_{i}^{-1} \mathbf{a}_{i}\right) .
\end{aligned}
$$

We can compute $\Delta t_{1}$ from Eq. (A.26), and $\Delta t_{2}$ from Eq. (A.27) using the threshold condition on $v$, we get

$$
\begin{aligned}
E_{v}= & \frac{R_{v}}{\lambda_{2}-\lambda_{1}}\left(k\left(V_{\mathrm{rev}}-V_{Q}\right)+E_{v}+l\right) \exp \left(\lambda_{2} \Delta t_{1}\right)\left(1-\exp \left(\left(\lambda_{2}+R_{v}\right) T\right)\right) \\
& +\left(\frac{\lambda_{1}}{\lambda_{2}-\lambda_{1}}\right) u_{0} \exp \left(\lambda_{1}\left(\Delta t_{1}+T+\Delta+\Delta t\right)\right) \\
& -\left(\frac{R_{v}}{\lambda_{2}-\lambda_{1}}\right) u_{0}\left(\exp \left(\lambda_{1}(\Delta+\Delta t)+\lambda_{2} \Delta t_{1}\right)\right)\left(\exp \left(\lambda_{1} T\right)-\exp \left(\left(\lambda_{2}+R_{v}\right) T\right)\right) . \\
E_{v}= & \frac{R_{v}}{\lambda_{2}-\lambda_{1}}\left(k\left(V_{\mathrm{rev}}-V_{Q}\right)+E_{v}+l\right) \exp \left(\lambda_{2} \Delta t_{2}\right)\left(1-\exp \left(\left(\lambda_{2}+R_{v}\right) T\right)\right) \\
& +\left(\frac{\lambda_{1}}{\lambda_{2}-\lambda_{1}}\right) u_{0} \exp \left(\lambda_{1}\left(\Delta t_{2}+T+\Delta-\Delta t\right)\right) \\
& -\left(\frac{R_{v}}{\lambda_{2}-\lambda_{1}}\right) u_{0}\left(\exp \left(\lambda_{1}(\Delta-\Delta t)+\lambda_{2} \Delta t_{2}\right)\right)\left(\exp \left(\lambda_{1} T\right)-\exp \left(\left(\lambda_{2}+R_{v}\right) T\right)\right) .
\end{aligned}
$$

These two equations, (A.29) and (A.30), can be solved numerically, and using Eq. (11) we can compute the synchronization rate $\sigma_{\mathrm{s}}$. 
Appendix B. Calculation of the synchronization rate in the piecewise linear model for a coupling on the $v$ and $u$ components

\section{B.1. Constant coupling}

We now have to evaluate the RHS of Eqs. (A.11) and (A.12) with the new coupling terms defined by Eq. (14):

$$
\mathbf{M}^{-1}\left(\mathbf{a}_{\mathbf{f}}-\mathbf{a}_{\mathbf{i}}\right)=\left(\begin{array}{c}
I_{u} / b \\
I_{u} / k b-I_{v} / k
\end{array}\right) .
$$

Since $\lambda_{2}<0$ and $\lambda_{1}<0$, with $\lambda_{1} / \lambda_{2}=-b c / \lambda_{2} \ll 1$, and since the first component of Eq. (B.1) is now different from zero, we can neglect, for the non-vanishing values of $t, \exp \left(\lambda_{2} t\right)$ compared with $\exp \left(\lambda_{1} t\right)$. Hence,

$$
\mathbf{C} \exp (\mathbf{D} t) \mathbf{C}^{-1}=\left(\begin{array}{cc}
\exp \left(\lambda_{1} t\right) & 0 \\
\exp \left(\lambda_{1}\right) /\left(\lambda_{2}-\lambda_{1}\right) & 0
\end{array}\right) .
$$

We can calculate $\Delta t_{1}$ from Eq. (A.11), using the threshold condition on $v$, namely $v_{1}\left(t_{2}-\Delta+T+\Delta t_{1}\right)=V_{\text {th }}$. Then, with $\tilde{V}=E_{v}\left(\lambda_{2}-\lambda_{1}\right) / \lambda_{2}$, we get

$$
\tilde{V}=\exp \left(\lambda_{1} \Delta t_{1}\right)\left(\left(I_{u} / b\right)\left(1-\exp \left(\lambda_{1} T\right)\right)+u_{0} \exp \left(\lambda_{1}(\Delta t+T+\Delta)\right)\right) .
$$

Similarly we can obtain $\Delta t_{2}$ from Eq. (A.11):

$$
\tilde{V}=\exp \left(\lambda_{1} \Delta t_{2}\right)\left(\left(I_{u} / b\right)\left(1-\exp \left(\lambda_{1} T\right)\right)+u_{0} \exp \left(\lambda_{1}(-\Delta t+T+\Delta)\right)\right) .
$$

Using Eqs. (11), (B.3) and (B.4), we finally obtain:

$$
\sigma_{\mathrm{s}}=\frac{\Delta t_{2}-\Delta t_{1}}{\Delta t}-1
$$

where

$$
\Delta t_{2}-\Delta t_{1}=\frac{1}{\lambda_{1}} \ln \left(\frac{J+u_{0} \exp \left(\lambda_{1}(\Delta t+T+\Delta)\right)}{J+u_{0} \exp \left(\lambda_{1}(-\Delta t+T+\Delta)\right)}\right)
$$

and

$$
J=\frac{I_{u}}{b}\left(1-\exp \left(\lambda_{1} T\right)\right)
$$

\section{B.2. Coupling with a linear dependency on postsynaptic potential}

During inhibition, we have the following system:

$$
\begin{aligned}
& \dot{v}=k\left(v-V_{Q}\right)+l-u+\left(v-V_{\mathrm{rev}}\right) R_{v}+E_{v}, \\
& \dot{u}=-c\left(b u-\left(v-V_{\mathrm{rev}}\right) R_{u}\right) .
\end{aligned}
$$

So as to avoid the firing during inhibition, one must have $V_{\mathrm{rev}}<V_{\mathrm{th}}-E_{v} /\left(R_{u} / b-R_{v}\right)$. Using the threshold condition on variable $v$ for neuron 1 , we get

$$
\begin{aligned}
E_{v}= & \exp \left(\lambda_{1} \Delta t_{1}\right)\left(\frac{-R_{u}\left(k\left(V_{\mathrm{rev}}-V_{Q}\right)+l+E_{v}\right)\left(1-\exp \left(\left(\lambda_{1}+\delta_{1}\right) T\right)\right)}{b\left(k+R_{v}-R_{u} / b\right)}\right. \\
& \left.+u_{0} \exp \left(\lambda_{1}(T+\Delta+\Delta t)\right) \exp \left(\delta_{1} T\right)\right)
\end{aligned}
$$


In the same way, for neuron 2 , we get

$$
\begin{aligned}
E_{v}= & \exp \left(\lambda_{1} \Delta t_{2}\right)\left(\frac{-R_{u}\left(k\left(V_{\text {rev }}-V_{Q}\right)+l+E_{v}\right)\left(1-\exp \left(\left(\lambda_{1}+\delta_{1}\right) T\right)\right)}{b\left(k+R_{v}-R_{u} / b\right)}\right. \\
& \left.+u_{0} \exp \left(\lambda_{1}(T+\Delta-\Delta t)\right) \exp \left(\delta_{1} T\right)\right)
\end{aligned}
$$

Using these two equations and Eq. (11) we can calculate analytically the synchronization rate $\sigma_{\mathrm{s}}$, we get:

$$
\sigma_{\mathrm{s}}=\frac{1}{\lambda_{1} \Delta t} \ln \left(\frac{\tilde{J}+u_{0} \exp \left(\lambda_{1}(\Delta t+T+\Delta)+\delta_{1} T\right)}{\tilde{J}+u_{0} \exp \left(\lambda_{1}(-\Delta t+T+\Delta)+\delta_{1} T\right)}\right)-1
$$

with

$$
\tilde{J}=\frac{-R_{u}\left(k\left(V_{\mathrm{rev}}-V_{Q}\right)+l+E_{v}\right)\left(1-\exp \left(\left(\lambda_{1}+\delta_{1}\right) T\right)\right)}{b\left(k+R_{v}-R_{u} / b\right)} .
$$

\section{References}

[1] L.F. Abbott and T.B. Kepler, Model neurons: From Hodgkin-Huxley to Hopfield, in: Statistical Mechanics of Neural Networks, ed. L. Garrido (Springer, Berlin, 1990).

[2] A.P. Andersen, J.C. Eccles and Y. Loyning, Recurrent inhibition in the Hippocampus with identification of the inhibitory cell and its synapses, Nature 198 (1963) 540-542.

[3] A.P. Andersen, J.C. Eccles and Y. Loyning, Pathway of postsynaptic inhibition in the Hippocampus, J. Neurophysiology 27 (1964) 608-619.

[4] E. Av-Ron, H. Parnas and L.A. Segel, A minimal biophysical model for excitable and oscillatory neuron, Biol. Cybernet. 65 (1991) 487-500.

[5] D.L. Benson and P.A. Cohen, Activity-independent segregation of excitatory and inhibitory synaptic terminals in cultured hippocampal neurons, J. Neurosci. 16 (1996) 6414-6432.

[6] T.W. Blackstad and P.R. Flood, Ultrastructure of hippocampal axo-somatic synapses, Nature 198 (1963) 542-543.

[7] V. Braitenberg and A. Schuz, Anatomy of the Cortex: Statistics and Geometry (Springer, Berlin, 1991).

[8] G. Buzsáki and J.J. Chrobak, Temporal structure in spatially organized neuronal ensembles: a role for interneuronal networks, Current Biology 5 (1995) 504-510.

[9] C.E. Carr and M. Konishi, Axonal delay line for time measurement in the owl's brainstem, Proc. Nat. Academy Sci. 85 (1988) $8311-8315$.

[10] A. Destexhe, Z. Mainen and T.J. Sejnowski, Synaptic currents, neuromodulation and kinetics models, in: The Handbook of Brain Theory and Neural Networks, ed. A. Arbib (MIT Press, Cambridge, MA, 1995) pp. 956-959.

[11] U. Ernst, K. Pawelzik and T. Geisel, Synchronization induced by temporal delays in pulse-coupled oscillators, Phys. Rev. Lett. 74 (1995) 1570-1573.

[12] W. Gerstner, R. Ritz and J. Leo van Hemmen, Why spikes? Hebbian learning and retrieval of time resolved excitation patterns, Biol. Cybernet. 69 (1993) 503-515.

[13] J. Grasman, Asymptotic Methods for Relaxation Oscillations and Applications (Springer, Berlin, 1987).

[14] E.G. Gray, Axo-somatic and axo-dendritic synapses of the cerebral cortex: An electron microscope study, J. Anat. 93 (1959) $420-433$.

[15] L.B. Haberly, Summed potentials evoked in opossum prepyriform cortex, J. Neurophysiology 36 (1973) 775-788.

[16] C. Hammond and D. Trisch, Neurobiologie Cellulaire (Doin, Paris, 1990).

[17] D. Hansel, G. Mato and C. Meunier, Phase dynamics for weakly coupled Hodgkin-Huxley neurons, Europhys. Lett. 23 (1993) 369-372.

[18] D. Hansel, G. Mato and C. Meunier, Synchrony in excitatory neural networks, Neural Comput. 7 (1995) 307-337.

[19] A.L. Hodgkin and A.F. Huxley, A quantitative description of membrane current and its application to conduction and excitation in nerve, J. Physiol. 117 (1952) 500-544.

[20] E.R. Kandel and J.H. Schwartz, Directly gated transmission at central synapses, in: Principles of Neural Science, eds. E.R. Kandel, J.H. Schwartz and T.M. Jessell, Ch. 11 (Prentice-Hall, London, 1991) pp. 153-172.

[21] E.R. Kandel, J.H. Schwartz and T.M. Jessell, Principles of Neural Science, 3rd Ed. (Prentice-Hall, London, 1991).

[22] T.B. Kepler, L.F. Abbott and E. Marder, Reduction of conductance based neuron models, Biol. Cybernet. 66 (1992) $381-387$.

[23] J. Koester, Passive membrane properties of the neuron, in: Principles of Neural Science, eds. E.R. Kandel, J.H. Schwartz and T.M. Jessell, Ch. 7 (Prentice-Hall, London, 1991) pp. 96-103. 
[24] V.I. Krinskii and Y.M. Kokoz, Analysis of the equations of excitable membranes: I. Reduction of the Hodgkin-Huxley equations to a second order system, Biofizika 18 (1973) 506-511.

[25] R. Miles, R.D. Traub and R.K.S. Wong, Spread of synchronous firing in longitudinal slices from the CA3 region in the Hippocampus, J. Neurophysiology 60 (1988) 1471-1496.

[26] C. Morris and H. Lecar, Voltage oscillations in the barnacle giant muscle fiber, Biophys. Journal 35 (1981) 193-213.

[27] E. Niebur, H.G. Schuster and D.M. Kammen, Collective frequencies and metastability in networks of limit-cycle oscillators with time delays, Phys. Rev. Lett. 67 (1991) 2753-2756.

[28] A. Nischwitz and H. Glünder, Local lateral inhibition: A key to spike synchronization?, Biol. Cybernet. 73 (1995) 389-400.

[29] L.G. Nowak and J. Bullier, The timing of information transfer in the visual system, Cerebral Cortex 12 (1997) in press.

[30] R.E. Plant, Simple analogs for nerve membrane equations, in: Nonlinear Systems and Applications, ed. V. Lakshmikantham (Academic Press, New York, 1997) pp. 647-654.

[31] G. Renversez and O. Parodi, Synchronization in neural oscillators through local delayed inhibition, in: Spatiotemporal Models in Biological and Artificial Systems, eds. F.L. Silva, J.C. Principe and L.B. Almeida (IOS Press, Amsterdam, 1996) pp. 144-151.

[32] J. Rinzel and G.B. Ermentrout, Analysis of neural excitability and oscillations, in: Methods in Neuronal Modeling: From synapses to Networks, eds. C. Koch and I. Segev (MIT Press, Cambridge, MA, 1989) pp. 135-169.

[33] J. Rinzel and J.B. Keller, Traveling waves solutions of a nerve conduction equation, Biophys. J. 13 (1973) 1313-1337.

[34] M. Robertson and K.G. Pearson, Neural circuits in the flight system of the locust, J. Neurophysiology 53 (1985) 110-128.

[35] H.G. Schuster and P. Wagner, Mutual entrainment of two limit cycle oscillators with time delayed couplings, Prog. Theor. Phys. 81 (1989) 939-945.

[36] D. Somers and N. Kopell, Rapid synchronization through fast threshold modulation, Biol. Cybernet. 68 (1993) $393-407$.

[37] D. Somers and N. Kopell, Waves and synchrony in networks of oscillators of relaxation and non-relaxation type, Physica D 89 (1995) $169-183$.

[38] D. Terman and D. Wang, Global competition and local cooperation in a network of neural oscillators, Physica D 81 (1995) 148-176.

[39] S. Thorpe, Spike arrival times: A highly efficient coding scheme for neural networks, in: Parallel Processing in Neural Systems, eds. R. Eckmiller, G. Hartman and G. Hauske (Elsevier, Amsterdam, 1990) pp. 91-94.

[40] C. van Vreeswijk, L.F. Abbott and G.B. Ermentrout, When inhibition not excitation synchronizes neural firing, J. Comput. Neurosci. 1 (1994) 313-321.

[41] M.A. Whittington, R.D. Traub and J.G.R. Jefferys, Synchronized oscillations in interneuron networks driven by metabotropic glutamate receptor activation, Nature 373 (1995) 612-615. 